\title{
INTERSECTION PROPERTIES OF THE UNIT BALL
}

\author{
CARLO ALBERTO DE BERNARDI AND LIBOR VESELÝ
}

\begin{abstract}
Let $X$ be a real Banach space with the closed unit ball $B_{X}$ and the dual $X^{*}$. We say that $X$ has the intersection property (I) (general intersection property (GI), respectively) if, for each countable family (for each family, respectively) $\left\{B_{i}\right\}_{i \in A}$ of equivalent closed unit balls such that $B_{X}=\bigcap_{i \in A} B_{i}$, one has $B_{X^{* *}}=\bigcap_{i \in A} B_{i}^{\circ \circ}$, where $B_{i}^{\circ \circ}$ is the bipolar set of $B_{i}$, that is, the bidual unit ball corresponding to $B_{i}$. In this paper we study relations between properties (I) and (GI), and geometric and differentiability properties of $X$. For example, it follows by our results that if $X$ is Fréchet smooth or $X$ is a polyhedral Banach space then $X$ satisfies property (GI), and hence also property (I). Moreover, for separable spaces $X$, properties (I) and (GI) are equivalent and they imply that $X$ has the ball generated property. However, properties (I) and (GI) are not equivalent in general. One of our main results concerns $C(K)$ spaces: under certain topological condition on $K$, satisfied for example by all zerodimensional compact spaces and hence by all scattered compact spaces, we prove that $C(K)$ satisfies (I) if and only if every nonempty $G_{\delta}$-subset of $K$ has nonempty interior.
\end{abstract}

\section{INTRODUCTION}

Let $X=(X,\|\cdot\|)$ be a real Banach space and $B_{X}$ its closed unit ball.

Assuming that the norm of $X$ coincides with the (pointwise) supremum of a family of equivalent norms $\|\cdot\|_{i}(i \in A)$, it is natural to ask whether also the norm of the bidual $X^{* *}$ is the supremum of the corresponding family of bidual norms.

Clearly, the same question can be reformulated in terms of closed unit balls: for each $i \in A$, if $B_{i}$ is the closed unit ball of the norm $\|\cdot\|_{i}$, and $B_{i}^{\circ \circ}$ is the bipolar set of $B_{i}$ (that is, the corresponding bidual closed unit ball), does the following implication hold?

$$
B_{X}=\bigcap_{i \in A} B_{i} \Rightarrow B_{X^{* *}}=\bigcap_{i \in A} B_{i}^{\circ} .
$$

2010 Mathematics Subject Classification. Primary 46B20; Secondary 46B04, 46B10, $46 \mathrm{~B} 25$.

Key words and phrases. Geometry of Banach spaces, sequences of equivalent norms, space of continuous functions, reflexivity. 
If the answer is affirmative for each countable family (for each family, respectively) $\left\{\|\cdot\|_{i}\right\}_{i \in A}$ of equivalent norms on $X$, we say that $X$ has the intersection property (I) (has the general intersection property (GI), respectively).

The aim of the present paper is to study relations between properties (I) and (GI), and geometric and differentiability properties of Banach spaces.

After some preliminary results, the first step in our study consists in dual characterizations of property (I), given in Proposition 3.3, including a characterization in terms of the so-called intermediate envelope (defined in [8]; see the text after Definition 2.7). These characterizations enable us to prove all main results of the paper concerning property (I). Then we present a similar characterization for property (GI) and we study relations between the two properties: it is clear that property (GI) implies property (I), and Theorem 3.6 shows that the two properties coincide if $X$ is separable or $w^{*}$-sequentially dense in its bidual. However, properties (I) and (GI) are not equivalent in general (see Propositions 5.8 and 5.1).

It is an obvious observation that if $X$ is reflexive then every equivalent norm on $X$ has property (I). In Section 4, we give several characterizations of reflexivity in terms of properties (I) and (GI). In particular, Theorem 4.1 shows that also the converse of the above observation holds true.

In Section 5, we study properties (I) and (GI) in $C(K)$ spaces and in some other classical spaces. In Corollary 5.4, one of the main results of our paper, we prove that under a topological condition on $K$ (satisfied for example by all zero-dimensional compact spaces and hence also by all scattered compact spaces), the space $C(K)$ satisfies (I) if and only if every nonempty $G_{\delta}$-subset of $K$ has nonempty interior. As for property (GI), its characterization for $C(K)$ spaces is much simpler: $C(K)$ has property (GI) if and only if $K$ is finite (see Proposition 5.1).

In Section 6, we present some results concerning relations between property (GI), the ball generated property, nicely smoothness and some other geometrical properties of the dual unit ball. Theorem 6.6 collects the main results in this direction, and its proof relies on the techniques introduced in [5, 4]. As a corollary, we obtain for example that a Banach space $X$ has property (GI), and hence property (I), whenever $X$ is Fréchet smooth or $X$ has the Mazur intersection property or $X$ is a polyhedral Banach space. Moreover, at the end of the section we study the problem of finding an equivalent norm on an Asplund space satisfying property (I). It turns out that the situation is analogous to that of the Mazur intersection property, and it depends on set-theoretic axioms (cf. [13, 1]).

We point out that some of our results from Sections 5 and 6 , are in some sense inspired by the paper [11] that contains results about a sequential version of the ball generated property, the so-called property $(\mathrm{P})$, some of which are formally similar to our results about property (I). Despite this, the relation 
between these two properties is not completely clear. It follows from Theorem 6.6 that in separable Banach spaces property (I) implies (P), however we do not know whether this implication holds for general Banach spaces (see Problem 8.4).

We conclude the paper with a section devoted to the study of a property called by us the union property (U). This property is defined in a similar way as property (I), by considering the (pointwise) infimum, instead of the supremum, of monotone sequences of equivalent norms. Similarly to property (I), property (U) can be reformulated using closed unit balls (see Definition 7.1). Proposition 7.2 provides several dual characterizations of property (U). As a corollary we obtain that if $X$ has (U) then $X$ is a Grothendieck space (Corollary 7.5); however the converse is not true (cf. Theorem 7.6). Proposition 7.4 characterizes property $(\mathrm{U})$ in terms of intermediate envelope as well as of the so-called 1-Grothendieck property (a quantitative version of the Grothendieck property, introduced in [2]). Combining our characterization and the results in [14], we obtain that $\ell_{\infty}$ has property (U). Moreover, by a characterization of reflexivity in terms of property (U), we provide an alternative proof of a result of O.F.K. Kalenda contained in [15] (see Remark 8.6).

\section{NotATIONS AND PRELIMINARIES}

In what follows, all (normed) linear spaces are real, and all topological spaces are Hausdorff. If not otherwise specified, the topological notions, like closure, interior etcetera, in a normed linear space are intended in the norm topology.

If $X=(X,\|\cdot\|)$ is a normed linear space then $X^{*}=\left(X^{*},\|\cdot\|\right)$ is its dual Banach space (equipped with the standard dual norm); $B_{X}, U_{X}$ and $S_{X}$ are the closed unit ball, the open unit ball and the unit sphere of $X$, respectively. The distance of a point $x \in X$ from a nonempty set $A \subset X$ is defined as $d(x, A):=\inf \{\|x-a\|: a \in A\}$. As usual, $X$ will sometimes be considered as a subspace of its second dual $X^{* *}$. With this in mind, given $x \in X$ and $x^{*} \in X^{*}$ we can write indifferently $x^{*} x$ or $x x^{*}$ to denote the value of $x^{*}$ at $x$.

The polar of a set $A \subset X$ is the set $A^{\circ}:=\left\{x^{*} \in X^{*}: \sup x^{*}(A) \leq 1\right\}$. For $B \subset X^{*}$, in addition to its polar $B^{\circ} \subset X^{* *}$ we can consider also the prepolar ${ }^{\circ} B=\{x \in X: \sup x(B) \leq 1\}=B^{\circ} \cap X$. It is obvious that these polar operations are inverse monotone with respect to the inclusion. Moreover, $A^{\circ}=(\overline{\operatorname{conv}} A)^{\circ}$, and ${ }^{\circ} B={ }^{\circ}\left(\overline{\operatorname{conv}}^{w^{*}} B\right)$. The following fact is well known.

Fact 2.1. Let $A \subset X$ and $B \subset X^{*}$ be convex sets containing the origin.

(a) ${ }^{\circ}\left(A^{\circ}\right)=\bar{A}, \quad\left({ }^{\circ} B\right)^{\circ}=\bar{B}^{w^{*}},\left(A^{\circ}\right)^{\circ}=\bar{A}^{w^{*}}\left(w^{*}\right.$-closure in $\left.X^{* *}\right)$.

(b) $A, A^{\circ}, B,{ }^{\circ} B$ is bounded if and only if the origin is an interior point of $A^{\circ}, \bar{A},{ }^{\circ} B, \bar{B}^{w^{*}}$, respectively. 
The set $B$ is said to be 1 -norming if $\|x\|=\sup x(B)$ for each $x \in X$. It is easy to see that:

$$
B \text { is 1-norming } \Longleftrightarrow \overline{\mathrm{conv}}^{w^{*}} B=B_{X^{*}} \Longleftrightarrow B \subset B_{X^{*}},{ }^{\circ} B=B_{X} .
$$

We shall also need some simple auxiliary facts. The first one is quite standard; we give here a proof for the sake of completeness.

Lemma 2.2. Let $X$ be a normed linear space, and let $A_{\alpha}(\alpha \in I)$ be bounded, closed, convex sets in $X$, all containing the origin.

(a) $\left[\bigcup_{\alpha \in I} A_{\alpha}\right]^{\circ}=\bigcap_{\alpha \in I} A_{\alpha}^{\circ}$.

(b) If $0 \in \operatorname{int}\left(\bigcap_{\alpha \in I} A_{\alpha}\right)$, then $\left(\bigcap_{\alpha \in I} A_{\alpha}\right)^{\circ}=\overline{\operatorname{conv}^{w}}\left(\bigcup_{\alpha \in I} A_{\alpha}^{\circ}\right)$.

Proof.

(a) $x^{*} \in\left[\bigcup_{\alpha} A_{\alpha}\right]^{\circ}$ if and only if $1 \geq \sup x^{*}\left(\bigcup_{\alpha} A_{\alpha}\right)=\sup _{\alpha} \sup x^{*}\left(A_{\alpha}\right)$, and this holds if and only if $x^{*} \in \bigcap_{\alpha \in I} A_{\alpha}^{\circ}$.

(b) For each $\beta \in I, A_{\beta}^{\circ} \subset\left(\bigcap_{\alpha} A_{\alpha}\right)^{\circ}$ (by inverse monotonicity of the polar), and hence

$$
\overline{\operatorname{conv}}^{w^{*}}\left(\bigcup_{\alpha \in I} A_{\alpha}^{\circ}\right) \subset\left(\bigcap_{\alpha \in I} A_{\alpha}\right)^{\circ} .
$$

By Fact 2.1, the right-hand side of (2) is bounded, and the left-hand side contains 0 in its interior and is $w^{*}$-compact. Assume that the inclusion in (2) is strict. By the Hahn-Banach separation theorem for the $w^{*}$-topology, there exists $x \in X \backslash\{0\}$ such that

(3) $1=\sup x\left(\overline{\operatorname{conv}}^{w^{*}}\left(\bigcup_{\alpha \in I} A_{\alpha}^{\circ}\right)\right)=\sup x\left(\bigcup_{\alpha \in I} A_{\alpha}^{\circ}\right)<\sup x\left(\left(\bigcap_{\alpha \in I} A_{\alpha}\right)^{\circ}\right)$.

The inequality in (3) implies that $x \notin{ }^{\circ}\left[\left(\bigcap_{\alpha} A_{\alpha}\right)^{\circ}\right]=\bigcap_{\alpha} A_{\alpha}$. On the other hand, the equalities in (3) together with (a) above give

$$
\begin{aligned}
x & { }^{\circ}\left(\bigcup_{\alpha} A_{\alpha}^{\circ}\right)=\left(\bigcup_{\alpha} A_{\alpha}^{\circ}\right)^{\circ} \cap X \\
& =\bigcap_{\alpha} A_{\alpha}^{\circ \circ} \cap X=\bigcap_{\alpha}{ }^{\circ}\left(A_{\alpha}^{\circ}\right)=\bigcap_{\alpha} A_{\alpha} .
\end{aligned}
$$

This contradiction completes the proof.

Fact 2.3. Let $K_{1}, \ldots K_{n}$ be compact convex sets in a (Hausdorff) topological vector space. Then the sets $\operatorname{conv}\left(K_{1} \cup \cdots \cup K_{n}\right)$ and $K_{1}+\ldots+K_{n}$ are compact, and hence closed. (This is well known.)

Fact 2.4. Let $C$ be a convex set in a normed linear space, and int $C \neq \emptyset$. Then $\operatorname{int}(\bar{C})=\operatorname{int} C . \quad($ See $[16,(4.6)]$.

Notation 2.5. Given sets $E, E_{1}, E_{2} \ldots$ in a normed linear space, we shall use the following, quite intuitive notation:

(a) $E_{n} \nearrow E$ means that the sequence $\left\{E_{n}\right\}$ is increasing and its union is $E$;

(b) $E_{n} \bar{\nearrow} E$ means that the sequence $\left\{E_{n}\right\}$ is increasing and the closure of its union is $E$; 
(c) $E_{n} \searrow E$ means that the sequence $\left\{E_{n}\right\}$ is decreasing and its intersection is $E$.

Lemma 2.6. Let $X$ be a normed linear space. Suppose that $\left\{C_{n}\right\}$ is an increasing sequence of convex subsets of $B_{X}$ such that $0 \in \operatorname{int} C_{1}$. Then the following are equivalent.

(i) $C_{n} \bar{\nearrow} B_{X}$;

(ii) $\bigcup_{n \in \mathbb{N}} C_{n} \supset U_{X}$;

(iii) $C_{n}^{\circ} \searrow B_{X^{*}}$.

Proof. Let us denote $C:=\bigcup_{n} C_{n}$. The implication $(i i) \Rightarrow(i)$ is obvious. To prove that $(i) \Rightarrow($ iii $)$, we can use Lemma 2.2: $B_{X^{*}}=\left(B_{X}\right)^{\circ}=C^{\circ}=\bigcap_{n} C_{n}^{\circ}$. Finally, assume (iii). Then $U_{X}=\operatorname{int} B_{X}=\operatorname{int}(\bar{C})=\operatorname{int} C$, where the last equality holds by Fact 2.4.

Definition 2.7. Let $K$ be a $w^{*}$-compact convex subset of a dual Banach space $X^{*}$ and $E \subset K$, we say that the set $E(I)$-generates $K$ iff the following condition holds:

if $E \subset \bigcup_{1}^{\infty} C_{n}$ where the sets $C_{n} \subset K$ are $w^{*}$-compact and convex, then $K=\overline{\operatorname{conv}}\left(\bigcup_{1}^{\infty} C_{n}\right)$ (norm-closure!).

It is easy to see, that the set $E(I)$-generates $K$ if the set $K$ coincides with the so-called intermediate envelope of $E$, i.e., the set given by

$$
(\mathrm{I})-\operatorname{env}(E):=\bigcap\left\{\overline{\bigcup_{n \in \mathbb{N}} \overline{\operatorname{conv}} w^{*} E_{n}}: E_{n} \nearrow E\right\} .
$$

This notion was introduced by V.P. Fonf and J. Lindenstrauss in [8] and studied by several authors (see, e.g., $[2,14,15,18]$ and the references therein). Let us recall the following so-called Boundary Theorem (for a relatively simple proof, see [18, Theorem 2]).

Theorem 2.8 (Boundary Theorem). Let $X$ be a Banach space, $K \subset X^{*} a$ $w^{*}$-compact convex set, and $\mathcal{B} \subset K$ a boundary for $K$ (that is, for each $x \in X$ there exists $b^{*} \in \mathcal{B}$ such that $\left.x b^{*}=\sup x(K)\right)$. Then $\mathcal{B}(\mathrm{I})$-generates $K$.

Notice that the above theorem can be reformulated in the following form: if the boundary $\mathcal{B}$ is covered by a countable union of $w^{*}$-compact convex sets, then $K$ is contained in the norm-closed convex hull of that union.

We shall need the following result concerning the intermediate envelope of a norm-separable set (see [14, Remark 1.1(ii)] or [8, Proposition 2.2(a)]).

Lemma 2.9. Let $X$ be a Banach space and let $E \subset X^{*}$. If $E$ is norm-separable then

$$
(\mathrm{I})-\operatorname{env}(E)=\overline{\operatorname{conv}}(E) \text {. }
$$




\section{INTERSECTION PROPERTIES}

Let $X$ be a normed linear space. By an equivalent ball in $X$ we mean a set $B \subset X$ that coincides with the closed unit ball of an equivalent norm on $X$. Clearly, such sets are exactly the closed, bounded, symmetric convex sets with nonempty interior. Let us denote by $\mathcal{E B}(X)$ the set of all equivalent balls in $X$.

Definition 3.1. We shall say that a normed linear space $X$ :

(a) has property (I) if

$$
\left\{B_{n}\right\}_{n \in \mathbb{N}} \subset \mathcal{E B}(X), \bigcap_{n} B_{n}=B_{X} \Rightarrow \bigcap_{n} B_{n}^{\circ \circ}=B_{X^{* *}} .
$$

(b) has property (GI) if

$$
\left\{B_{\alpha}\right\}_{\alpha \in I} \subset \mathcal{E B}(X), \bigcap_{\alpha} B_{\alpha}=B_{X} \Rightarrow \bigcap_{\alpha} B_{\alpha}^{\circ \circ}=B_{X^{* *}} .
$$

("(I)" comes from "intersection"; "(GI)" comes from "general intersection".)

Observation 3.2. It is easy to verify that $X$ has property (I) if and only if the implication (4) holds for all equi-bounded sequences $\left\{B_{n}\right\}$, if and only if (4) holds for all decreasing sequences $\left\{B_{n}\right\}$. (It suffices to consider the sets $C_{n}:=\bigcap_{k<n} B_{k}$.)

And analogously, $X$ has property (GI) if and only if (5) holds for all equibounded families $\left\{B_{\alpha}\right\}$. (Fix some $\alpha_{0} \in I$, and consider $C_{\alpha}:=B_{\alpha} \cap B_{\alpha_{0}}$.)

Now we are ready to state some dual characterizations of properties (I) and (GI).

Proposition 3.3. For a normed linear space $X$ the following assertions are equivalent.

(i) $X$ has property (I).

(ii) For each sequence $\left\{D_{n}\right\} \subset \mathcal{E B}\left(X^{*}\right)$ such that each $D_{n}$ is $w^{*}$-compact and the set $D:=\operatorname{conv}\left(\bigcup_{n} D_{n}\right)$ is 1-norming, one has $\bar{D}=B_{X^{*}}$.

(iii) For each sequence $\left\{D_{n}\right\}$ of $w^{*}$-compact equivalent balls in $X^{*}$ such that $D_{n} \nearrow D$ and $D$ is 1-norming, one has $\bar{D}=B_{X^{*}}$.

(iv) For each sequence $\left\{C_{n}\right\}$ of $w^{*}$-compact, symmetric, convex sets in $X^{*}$ such that $C_{n} \nearrow C$ and $C$ is 1-norming, one has $\bar{C}=B_{X^{*}}$.

(v) For each symmetric 1-norming $E \subset X^{*}$ one has $(\mathrm{I})$-env $(E)=B_{X^{*}}$ (see Definition 2.7 and the text after it).

Proof. Several times without mentioning it, we shall use Lemma 2.2 and basic properties of polars.

$(i) \Rightarrow(i i)$. Let $\left\{D_{n}\right\}$ be as in (ii). Put $B_{n}:={ }^{\circ} D_{n}(n \in \mathbb{N})$, and observe that $B_{n}^{\circ}=D_{n}$ and $B_{n} \in \mathcal{E} \mathcal{B}(X)$. Moreover,

$$
\bigcap_{n} B_{n}={ }^{\circ}\left[\left(\bigcap_{n} B_{n}\right)^{\circ}\right]={ }^{\circ}\left[\overline{\operatorname{conv}}^{w^{*}}\left(\bigcup_{n} B_{n}^{\circ}\right)\right]={ }^{\circ}\left[\bar{D}^{w^{*}}\right]={ }^{\circ} B_{X^{*}}=B_{X} .
$$


By (i), we have $\bigcap_{n} B_{n}^{\circ \circ}=B_{X^{* *}}$. Consequently,

$$
\bar{D}={ }^{\circ}\left(D^{\circ}\right)={ }^{\circ}\left(\bigcap_{n} D_{n}^{\circ}\right)={ }^{\circ}\left(\bigcap_{n} B_{n}^{\circ \circ}\right)={ }^{\circ} B_{X^{* *}}=B_{X^{*}} .
$$

$(i i) \Rightarrow(i)$. Let $\left\{B_{n}\right\}$ be as in Definition 3.1(a). Then the sets $D_{n}:=B_{n}^{\circ}$ are $w^{*}$ compact elements of $\mathcal{E B}\left(X^{*}\right)$, and $\overline{\mathrm{conv}} w^{*}\left(\bigcup_{n} D_{n}\right)=\left(\bigcap_{n} B_{n}\right)^{\circ}=B_{X^{*}}$. Since $D:=\operatorname{conv}\left(\bigcup_{n} D_{n}\right)$ is 1-norming, we have by (ii) that $\bar{D}=B_{X^{*}}$. Consequently,

$$
\bigcap_{n} B_{n}^{\circ \circ}=\bigcap_{n} D_{n}^{\circ}=D^{\circ}=\left(B_{X^{*}}\right)^{\circ}=B_{X^{* *}} .
$$

The equivalence $(i) \Leftrightarrow$ (iii) follows by the same proof, taking into account Observation 3.2.

$($ iii $) \Rightarrow($ iv $)$. Let $\left\{C_{n}\right\}$ be as in (iv). We have to show that $\bar{C}=B_{X^{*}}$. Suppose on the contrary that there exist $\varepsilon>0$ and $x^{*} \in B_{X^{*}}$ such that $d\left(x^{*}, C\right)>2 \varepsilon$. Let us consider the $w^{*}$-compact equivalent balls

$$
D_{n}:=\left(C_{n}+\varepsilon B_{X^{*}}\right) \cap B_{X^{*}}, \quad n \in \mathbb{N} .
$$

The set $D:=\bigcup_{n} D_{n}$ is clearly 1-norming, hence by (iii) $x^{*} \in \bar{D}$. Since

$$
x^{*} \in \bar{D} \subset C+2 \varepsilon B_{X^{*}},
$$

we get a contradiction.

$(i v) \Rightarrow(v)$. Let $E \subset X^{*}$ be symmetric and 1-norming, and assume that $E \subset C$ where $C_{n} \nearrow C$ and the sets $C_{n} \subset B_{X^{*}}(n \in \mathbb{N})$ are $w^{*}$-compact and convex. Symmetry of $E$ and monotony of $\left\{C_{n}\right\}$ easily imply that $C_{n} \cap\left(-C_{n}\right) \nearrow C$. Since $C$ is clearly 1-norming, we have by (iv) that $B_{X^{*}}=\bar{C} \subset(\mathrm{I})$-env $(E) \subset$ $B_{X^{*}}$, and (v) follows.

Finally, the implication $(v) \Rightarrow($ iii $)$ follows directly from definitions.

Proposition 3.4. For a normed linear space $X$ the following assertions are equivalent.

(i) $X$ has property (GI).

(ii) For each 1-norming $D \in \mathcal{E} \mathcal{B}\left(X^{*}\right)$ one has $D=B_{X^{*}}$.

(iii) For each 1-norming symmetric set $E \subset X^{*}$ one has $\overline{c o n v} E=B_{X^{*}}$.

(iv) For each 1-norming set $E \subset X^{*}$ one has $\overline{\text { conv }} E=B_{X^{*}}$.

(v) For each $F \in X^{* *} \backslash B_{X^{* *}}$ one has $\operatorname{conv}\left(B_{X^{* *}} \cup\{ \pm F\}\right) \cap X \neq B_{X}$.

(vi) For each $F \in X^{* *} \backslash B_{X^{* *}}$ one has $\operatorname{conv}\left(B_{X^{* *}} \cup\{F\}\right) \cap X \neq B_{X}$.

Proof. Like in the previous proof, Lemma 2.2 will be widely used. We are going to prove the following implications:

$$
(i) \Rightarrow(i i) \Rightarrow(v) \Rightarrow(v i) \Rightarrow(i v) \Rightarrow(i i i) \Rightarrow(i) .
$$

$(i) \Rightarrow($ ii $)$. Let $D$ be as in (ii), and let $r \in(0,1)$ be such that $r B_{X^{*}} \subset D$. For $x^{*} \in D$, put

$$
A_{x^{*}}={ }^{\circ}\left\{ \pm x^{*}\right\} \cap \frac{1}{r} B_{X}={ }^{\circ}\left[-x^{*}, x^{*}\right] \cap \frac{1}{r} B_{X},
$$


(where $\left[-x^{*}, x^{*}\right]$ denotes the segment $\operatorname{conv}\left\{ \pm x^{*}\right\}$ ), and notice that $B_{X} \subset A_{x^{*}}$, and $A_{x^{*}}^{\circ}=\overline{\operatorname{conv}} w^{*}\left(\left[-x^{*}, x^{*}\right] \cup r B_{X^{*}}\right)=\operatorname{conv}\left(\left[-x^{*}, x^{*}\right] \cup r B_{X^{*}}\right)$ by Fact 2.3 . Hence

$$
\bigcap_{x^{*} \in D} A_{x^{*}}={ }^{\circ}\left[\left(\bigcap_{x^{*} \in D} A_{x^{*}}\right)^{\circ}\right]={ }^{\circ}\left[\overline{\operatorname{conv}^{w}}\left(\bigcup_{x^{*} \in D} A_{x^{*}}^{\circ}\right)\right]={ }^{\circ} D=B_{X} .
$$

By (i), $\bigcap_{x^{*} \in D} A_{x^{*}}^{\circ}=B_{X^{* *}}$. Therefore

$$
D={ }^{\circ}\left(D^{\circ}\right)={ }^{\circ}\left[\left(\bigcup_{x^{*} \in D} A_{x^{*}}^{\circ}\right)^{\circ}\right]={ }^{\circ}\left[\bigcap_{x^{*} \in D} A_{x^{*}}^{\circ}\right]=B_{X^{*}} .
$$

$(i i) \Rightarrow(v)$. Let $F \in X^{* *} \backslash B_{X^{* *}}$. Since $\|F\|>1$, the set $D:={ }^{\circ}\{ \pm F\} \cap B_{X^{*}}$ is an equivalent ball in $X^{*}$ and is properly contained in $B_{X^{*}}$. By (ii), $D$ is not 1-norming, and hence

$B_{X} \neq{ }^{\circ} D=D^{\circ} \cap X=\overline{\mathrm{conv}}^{w^{*}}\left(\{ \pm F\} \cup B_{X^{* *}}\right) \cap X=\operatorname{conv}\left(\{ \pm F\} \cup B_{X^{* *}}\right) \cap X$.

$(v) \Rightarrow(v i)$. Let $F \in X^{* *} \backslash B_{X^{* *}}$ and $\operatorname{conv}\left(\{ \pm F\} \cup B_{X^{* *}}\right) \cap X \supsetneqq B_{X}$. If $F \in X$, the inequality in (vi) is obvious. So let $F \notin X$. Fix some $x \in$ $\operatorname{conv}\left(\{ \pm F\} \cup B_{X^{* *}}\right) \cap X$ with $\|x\|>1$. Then we can write

$$
x=(\alpha-\beta) F+\gamma G,
$$

where $\alpha, \beta, \gamma \geq 0, \alpha+\beta+\gamma=1$, and $G \in B_{X^{* *}}$. Necessarily $\alpha \neq \beta$ and $\gamma>0$. First, let $\alpha>\beta$. Then $\alpha-\beta+\gamma>\gamma$, and we can write

$$
x=(\alpha-\beta+\gamma)\left[\frac{\alpha-\beta}{\alpha-\beta+\gamma} F+\frac{\gamma}{\alpha-\beta+\gamma} G\right] .
$$

Let us denote by $H$ the term in square brackets, and notice that $H$ belongs to $\operatorname{conv}\left(\{F\} \cup B_{X^{* *}}\right) \cap X$ and satisfies $\|H\|=\frac{1}{\alpha-\beta+\gamma}\|x\| \geq\|x\|>1$; thus the inequality in (vi) is satisfied. Finally, if $\alpha<\beta$ then we can proceed in the same way by considering $-x$ instead of $x$.

$(v i) \Rightarrow(i v)$. Let $E$ be as in (iv), $D=\overline{\mathrm{conv}} E$, and assume that $D \neq B_{X^{*}}$. By the Hahn-Banach separation theorem, there exists $F \in X^{* *}$ such that

$$
1=\sup F(D)<\sup F\left(B_{X^{*}}\right)=\|F\| .
$$

It follows that $D \subset{ }^{\circ}\{F\} \cap B_{X^{*}}$, and hence

$$
B_{X}={ }^{\circ} D=D^{\circ} \cap X \supset\left({ }^{\circ}\{F\} \cap B_{X^{*}}\right)^{\circ} \cap X=\operatorname{conv}\left(\{F\} \cup B_{X^{* *}}\right) \cap X \neq B_{X},
$$

which is a contradiction.

$(i v) \Rightarrow($ iii $)$ is obvious. It remains to show that $(i i i) \Rightarrow(i)$. Let $\left\{B_{\alpha}\right\}_{\alpha} \subset$ $\mathcal{E B}(X)$ be such that $\bigcap_{\alpha} B_{\alpha}=B_{X}$. Then $B_{X^{*}}=\left(\bigcap_{\alpha} B_{\alpha}\right)^{\circ}=\overline{\operatorname{conv}}^{w^{*}}\left(\bigcup_{\alpha} B_{\alpha}^{\circ}\right)$. This implies that the set $C:=\overline{\operatorname{conv}}\left(\bigcup_{\alpha} B_{\alpha}^{\circ}\right)$ is 1-norming. By (iii), we have $C=B_{X^{*}}$, and hence $\bigcap_{\alpha} B_{\alpha}^{\circ \circ}=C^{\circ}=B_{X^{* *}}$.

The following simple corollary shows that we can always restrict ourselves to Banach spaces. 
Corollary 3.5. Let $X$ be a normed linear space and $\hat{X}$ its completion, and let $\mathcal{I}$ denote one of properties (I) and (GI). Then $X$ has $\mathcal{I}$ if and only if $\hat{X}$ has $\mathcal{I}$.

Proof. This follows from the dual characterizations in Propositions 3.3 and 3.4 via the fact that $\hat{X}^{*}$ can be identified with $X^{*}$, and the $w^{*}$-topologies $\sigma\left(X^{*}, X\right)$ and $\sigma\left(X^{*}, \hat{X}\right)$ coincide on the dual ball $B_{X^{*}}$.

It is obvious that property (GI) implies property (I). The next theorem shows that the two properties are equivalent for some classes of Banach spaces. However, this equivalence is false for general Banach spaces, as we shall see in Section 5.

Theorem 3.6. Let $X$ be a Banach space with property (I). Suppose that at least one of the following conditions is satisfied:

(i) $\left(B_{X^{*}}, w^{*}\right)$ is hereditarily separable (e.g., $X$ is separable);

(ii) $X$ is $w^{*}$-sequentially dense in $X^{* *}$.

Then $X$ has property (GI). Moreover, if (i) is satisfied then $X^{*}$ is separable.

Proof. Let us prove that if $X$ has property (I) and satisfies (i) then $X$ has property (GI) and $X^{*}$ is separable. Let us consider a symmetric 1-norming set $D \subset B_{X^{*}}$, since $\left(B_{X^{*}}, w^{*}\right)$ is hereditarily separable, there exists a symmetric countable $w^{*}$-dense subset $B \subset D$. Clearly $B$ is 1-norming, and by Proposition $3.3(\mathrm{v})$ we have $(\mathrm{I})-\operatorname{env}(B)=B_{X^{*}}$. Since $B$ is countable, by Lemma 2.9 we have $(\mathrm{I})-\operatorname{env}(B)=\overline{\mathrm{conv}}(B)$ and hence

$$
\overline{\operatorname{conv}}(D) \subset B_{X^{*}}=(\mathrm{I})-\operatorname{env}(B)=\overline{\operatorname{conv}}(B) \subset \overline{\operatorname{conv}}(D) .
$$

In particular, we have $\overline{\mathrm{conv}}(B)=\overline{\mathrm{conv}}(D)=B_{X^{*}}$. By the arbitrariness of $D$ and by Proposition 3.4(iii), $X$ has property (GI). Moreover, since a set $D$ as above always exists and $B$ is countable, $X^{*}$ is separable.

Now let $X$ be $w^{*}$-sequentially dense in its bidual, and assume that $X$ fails (GI). There exists a 1-norming $D \in \mathcal{E B}\left(X^{*}\right)$ which is strictly contained in $B_{X^{*}}$. By the Hahn-Banach separation theorem, there exists $F \in X^{* *}$ such that $1=\sup F(D)<\sup F\left(B_{X^{*}}\right)=\|F\|$. The set $E:=\left\{x^{*} \in B_{X^{*}}:\left|F\left(x^{*}\right)\right| \leq 1\right\}$ is 1-norming since it contains $D$, however $E \neq B_{X^{*}}$. By our assumption, there exists a sequence $\left\{x_{k}\right\} \subset X \backslash B_{X^{* *}}=X \backslash B_{X}$ such that $x_{k} \stackrel{w^{*}}{\rightarrow} F$ in $X^{* *}$. The $w^{*}$-compact sets

$$
D_{n}:=\bigcap_{k \geq n}\left\{x^{*} \in B_{X^{*}}:\left|x^{*} x_{k}\right| \leq 1\right\}, \quad n \in \mathbb{N},
$$

form an increasing sequence in $\mathcal{E B}\left(X^{*}\right)$. Since the set $E_{0}:=\left\{x^{*} \in B_{X^{*}}\right.$ : $\left.\left|F\left(x^{*}\right)\right|<1\right\}$ is clearly norm dense in $E$, and $E_{0} \subset \bigcup_{n} D_{n} \subset E$, the set $C:=\bigcup_{n} D_{n}$ is 1-norming and satisfies $\bar{C}=E \neq B_{X^{*}}$. Thus $X$ fails (I). 
4. Characterizations of Reflexivity via properties (I), (GI)

Theorem 4.1. For a Banach space $X$, the following assertions are equivalent:

(i) $X$ is reflexive;

(ii) $X$ is a dual space and has property (GI);

(iii) every equivalent norm on $X$ has property (GI);

(iv) every equivalent norm on $X$ has property (I).

Proof. The implications $(i) \Rightarrow[(i i),(i i i),(i v)]$ and $(i i i) \Rightarrow(i v)$ are obvious.

(ii) $\Rightarrow(i)$. Assume that $X=Y^{*}$. Then $B_{Y}$ is a 1-norming subset of $Y^{* *}=X^{*}$. Proposition 3.4 implies that $B_{Y}=B_{Y^{* *}}$, and hence $Y$ is reflexive.

$(i v) \Rightarrow(i)$. Assume that $X$ is not reflexive, and fix some $0<\delta<\frac{1}{2}$. By a well-known James' result (see [17, Theorem 1.13.4]), there exist sequences $\left\{y_{n}^{*}\right\} \subset S_{X^{*}}$ and $\left\{y_{k}\right\} \subset S_{X}$ such that

$$
y_{n}^{*} y_{k} \begin{cases}=0 & \text { for } n>k \\ \geq 1-\delta & \text { for } n \leq k .\end{cases}
$$

Let $y^{*} \in B_{X^{*}}$ be a $w^{*}$-cluster point of $\left\{y_{n}^{*}\right\}_{n}$. Then we have

$$
y^{*} y_{k}=0, \quad k \in \mathbb{N} \text {. }
$$

Consider the $w^{*}$-closed (convex) sets

$$
D_{n}:=\left\{x^{*} \in 4 B_{X^{*}}:\left|x^{*} y_{j}\right| \leq \delta \text { for all } j \geq n\right\}, \quad n \in \mathbb{N} .
$$

These sets form an equi-bounded increasing sequence in $\mathcal{E B}\left(X^{*}\right)$. If we denote $D:=\bigcup_{n} D_{n}$, then $\bar{D}^{w^{*}}$ is the dual unit ball of an equivalent norm $\|\cdot\|$ on $X$, for which $D$ is 1 -norming. Let $F$ be a $w^{*}$-cluster point of $\left\{y_{k}\right\}_{k}$ in $X^{* *}$. Notice that $\sup F\left(D_{n}\right) \leq \delta$ for each $n$, and hence $\sup F(D) \leq \delta$. We have for each $n \geq 1$ that

$$
1-\delta \leq F\left(y_{n}^{*}\right) \leq 1
$$

Denote $z_{n}^{*}:=y_{1}^{*}-y_{n}^{*}(n \geq 2)$. Then (6) implies that $\left|z_{n}^{*} y_{k}\right| \leq \delta$ whenever $2 \leq n \leq k$, and hence $\left\{z_{n}^{*}\right\}_{2}^{\infty} \subset D$. Moreover, $u^{*}:=y_{1}^{*}-y^{*}$ is a $w^{*}$-cluster

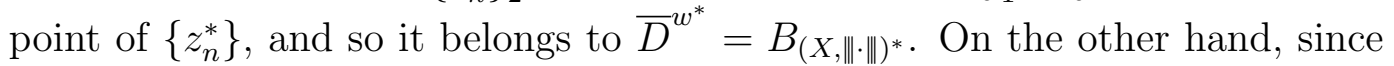
$F\left(y^{*}\right)=0$ by $(7)$, we obtain $F\left(u^{*}\right)=F\left(y_{1}^{*}\right) \geq 1-\delta>\delta \geq \sup F(D)$, and hence $u^{*} \notin \bar{D}$. By Proposition 3.3, (X,\| $\left.\cdot \|\right)$ fails to have (I).

Theorem 4.2. For a Banach space $X$, the following assertions are equivalent:

(i) $X$ is separable and reflexive;

(ii) $X$ is the dual of a separable Banach space, and satisfies (I).

Proof. Only the implication $(i i) \Rightarrow(i)$ requires a proof. Let $X=Y^{*}$ with $Y$ separable and assume that $X$ has property (I). By Proposition 3.3(v), we have (I)-env $\left(B_{Y}\right)=B_{Y^{* *}}$. Since $Y$ is separable, (I)-env $\left(B_{Y}\right)=B_{Y}$ by Lemma 2.9. Then $Y$ is moreover reflexive, and hence (i) is satisfied. 
It is not clear to us whether a nonreflexive dual space can have property (I) (see Problem 8.3).

\section{5. $C(K)$ SPACES, AND SOME OTHER CLASSICAL SPACES}

Given a compact (Hausdorff) topological space $K$, by $C(K)$ we mean the Banach space of all real-valued continuous functions on $K$, equipped with the supremum norm $\|\cdot\|_{\infty}$. As usual, every point $k \in K$ is then identifiable with the corresponding Dirac measure (or the evaluation functional) $\delta_{k} \in C(K)^{*}$. Using this identification, the topology of $K$ coincides with the $w^{*}$-topology of $C(K)^{*}$ (restricted to $\left.K \subset C(K)^{*}\right)$. We shall often consider $K$ as a subset of $C(K)^{*}$ without mentioning this.

Let us recall that a compact space $K$ is scattered if every its nonempty subset has an isolated point. The space $K$ is zero-dimensional if the family of its clopen sets is a base for the topology of $K$. It is well-known (see [7, Theorem 6.2.10]) that this is equivalent to say that $K$ is totally disconnected, that is, each of its nonempty connected subsets is a singleton. Hence every compact scattered space is zero-dimensional.

A pseudo-base of a topological space $T$ is a family of nonempty open sets such that every nonempty open subset of $T$ contains a member of the family. Every base is clearly a pseudo-base, but not vice-versa in general.

If $K$ is finite, then $C(K)$ is finite-dimensional (hence reflexive), and therefore $C(K)$ clearly has properties (GI) and (I). The next proposition shows that no other $C(K)$ spaces satisfy property (GI).

Proposition 5.1. Let $K$ be an infinite compact space. Then $C(K)$ fails property (GI). In particular, if $K$ is metrizable then $C(K)$ fails to have (I).

Proof. Let $p$ be an accumulation point of $K$, and consider the 1-norming set $E:=\{ \pm k: k \in K, k \neq p\}$ in $C(K)^{*}$. We claim that $p \notin \overline{\mathrm{conv}} E$. To show this, take an arbitrary $x^{*} \in$ conv $E$. Then we can write $x^{*}=\sum_{i=1}^{m}\left(\alpha_{i}-\beta_{i}\right) k_{i}$, where $k_{i} \in K \backslash\{p\}, \alpha_{i}, \beta_{i} \geq 0$, and $\sum_{i=1}^{m}\left(\alpha_{i}+\beta_{i}\right)=1$. There exists $x \in B_{C(K)}$ such that $x(p)=1$, and $x\left(k_{i}\right)=0$ for each $i=1, \ldots, m$. Then $\left\|p-x^{*}\right\| \geq$ $\left|x\left(p-x^{*}\right)\right|=1$. This shows that $d(p, \operatorname{conv} E) \geq 1$. Now the first statement holds by Proposition 3.4. The second statement follows from Theorem 3.6 since $C(K)$ is separable.

Now, let us study property (I) for $C(K)$ spaces. We shall see that the situation is quite different from that of (GI), and this will provide examples of spaces having (I) but not (GI).

Given a compact space $K$, let $K^{\prime}$ denote the set of its accumulation points, and let $G_{\delta}(K)$ be the set of its $G_{\delta}$-points (i.e., points $k \in K$ such that the singleton $\{k\}$ is a countable intersection of open sets). 
We shall need the following simple lemma.

Lemma 5.2. Let $V$ be a nonempty clopen subset of a compact space $K$, and $\varphi:=\mathbf{1}_{V}$ the characteristic function of $V$. Then $\varphi \in S_{C(K)}$, and the face

$$
F:=\left\{x^{*} \in B_{C(K)^{*}}: \varphi x^{*}=1\right\}
$$

of $B_{C(K)^{*}}$ is strongly exposed by $\varphi$ in the following sense:

$$
\forall \varepsilon>0, \exists \delta>0, \forall x^{*} \in B_{C(K)^{*}},\left(\varphi x^{*}>1-\delta \Rightarrow d\left(x^{*}, F\right)<\varepsilon\right) .
$$

Moreover, ext $F=V$.

Proof. It is obvious that $\varphi$ is continuous on $K$, and $\|\varphi\|_{\infty}=1$. Since it is well known that ext $B_{C(K)^{*}}=K \cup(-K)$, and since $F$ is an extremal set for $B_{C(K)^{*}}$, we have that ext $F=\left(\operatorname{ext} B_{C(K)^{*}}\right) \cap F=V$. Let us show that $F$ is a strongly exposed face of $B_{C(K)^{*}}$. Let $0<\delta<1$ and $x^{*} \in B_{C(K)^{*}}$ be such that $\varphi x^{*}>1-\delta$. Denoting $W:=K \backslash V$, we have (by the Krein-Milman theorem)

$$
x^{*} \in \overline{\mathrm{conv}} w^{*}( \pm K)=\operatorname{conv}\left[F \cup \overline{\operatorname{conv}} w^{*}(W \cup(-K))\right],
$$

and hence we can write $x^{*}=(1-\lambda) u^{*}+\lambda z^{*}$ where $0 \leq \lambda \leq 1, u^{*} \in F$, and $z^{*} \in \overline{\mathrm{conv}}^{w^{*}}(W \cup(-K))$. Since $\varphi z^{*} \leq 0$, we obtain that $1-\delta<\varphi x^{*} \leq 1-\lambda$, and hence $\lambda \leq \delta$. Consequently, $d\left(x^{*}, F\right) \leq\left\|x^{*}-u^{*}\right\|=\lambda\left\|z^{*}-u^{*}\right\| \leq 2 \delta$. We are done.

Theorem 5.3. Let $K$ be a compact space.

(a) If $C(K)$ has property (I), then every nonempty $G_{\delta}$-subset of $K$ has nonempty interior. In particular, $K^{\prime} \cap G_{\delta}(K)=\emptyset$.

(b) If every nonempty $G_{\delta}$-subset of $K$ contains a nonempty clopen set, then $C(K)$ has property (I).

Proof. (a) Assume that $K$ contains a nonempty $G_{\delta^{-}}$set with empty interior. Then its complement $L$ is a proper, dense $F_{\sigma}$-subset of $K$. Let us write $L=$ $\bigcup_{n \in \mathbb{N}} K_{n} \varsubsetneqq K$, where $\left\{K_{n}\right\}$ is an increasing sequence of closed sets in $K$. Then the sets

$$
C_{n}:=\overline{\operatorname{conv}}^{w^{*}}\left[K_{n} \cup\left(-K_{n}\right)\right], \quad n \in \mathbb{N},
$$

are $w^{*}$-compact, form an increasing sequence, and the set $C:=\bigcup_{n} C_{n}$ is 1-norming. However, for any $p \in K \backslash L$, the same type of proof as in Proposition 5.1 shows that $p \in B_{C(K)^{*}} \backslash \bar{C}$. Thus $C(K)$ fails to have (I) by Proposition 3.3. The last statement is now obvious.

(b) Let $\left\{D_{n}\right\} \subset \mathcal{E B}\left(C(K)^{*}\right)$ be an increasing sequence of $w^{*}$-compact sets such that $D:=\bigcup_{n} D_{n}$ is 1-norming. By Proposition 3.3, it suffices to show that $\bar{D}=B_{C(K)^{*}}$.

First, assume there exists $k_{0} \in K \backslash \bar{D}$. Fix $\varepsilon>0$ so that $d\left(k_{0}, D\right)>2 \varepsilon$. Then for each $n$ there exists $x_{n} \in S_{C(K)}$ such that

$$
x_{n}\left(k_{0}\right)>\max x_{n}\left(D_{n}\right)+2 \varepsilon \text {. }
$$


Since the sets $U_{n}:=\left\{k \in K \backslash D_{n}:\left|x_{n}(k)-x_{n}\left(k_{0}\right)\right|<\varepsilon\right\}$ are open neighborhoods of $k_{0}$ in $K$, the $G_{\delta^{-}}$set $U:=\bigcap_{n \in \mathbb{N}} U_{n}$ contains a nonempty clopen set $V$. By Lemma 5.2, the characteristic function $\varphi:=\mathbf{1}_{V} \in S_{C(K)}$ strongly exposes the face $F:=\varphi^{-1}(1) \cap B_{C(K)^{*}}$ of $B_{C(K)^{*}}$. Consider the corresponding $\delta>0$ for "our" $\varepsilon$ by that lemma. Since $D$ is $w^{*}$-dense in $B_{C(K)^{*}}$, there exist $m \in \mathbb{N}$ and $d^{*} \in D_{m}$ with $\varphi\left(d^{*}\right)>1-\delta$. Fix $u^{*} \in F$ with $\left\|d^{*}-u^{*}\right\|<\varepsilon$. Since the function $y^{*} \mapsto\left|x_{m} y^{*}-x_{m}\left(k_{0}\right)\right|$ is convex and $w^{*}$-continuous on $C(K)^{*}$, and less or equal to $\varepsilon$ on $V=\operatorname{ext} F$, we must have $\left|x_{m} u^{*}-x_{m}\left(k_{0}\right)\right| \leq \varepsilon$ by the Krein-Milman theorem. But then we obtain

$$
x_{m}\left(k_{0}\right) \leq x_{m} u^{*}+\varepsilon<x_{m} d^{*}+2 \varepsilon \leq \max x_{m}\left(D_{m}\right)+2 \varepsilon,
$$

a contradiction with (8).

So we must have $K \subset \bar{D}$. Let $\alpha>0$. The boundary $\mathcal{B}=K \cup(-K)$ for $B_{C(K)^{*}}$ is contained in $D+\alpha B_{C(K)^{*}}=\bigcup_{1}^{\infty}\left(D_{n}+\alpha B_{C(K)^{*}}\right)$. By the Boundary Theorem (Theorem 2.8 and the text after it),

$$
B_{C(K)^{*}} \subset \overline{\operatorname{conv}}\left[\bigcup_{n}\left(D_{n}+\alpha B_{C(K)^{*}}\right)\right] \subset D+2 \alpha B_{C(K)^{*}} .
$$

Since $\alpha>0$ was arbitrary, we conclude that $\bar{D}=B_{C(K)^{*}}$. We are done.

Corollary 5.4. For a compact space $K$, let us consider the following three conditions:

(i) $C(K)$ has property (I);

(ii) every nonempty $G_{\delta}$-set in $K$ has nonempty interior;

(iii) $K^{\prime} \cap G_{\delta}(K)=\emptyset$.

Now the following assertions hold.

(a) If nonempty clopen sets form a pseudo-base for $K$ (e.g., if $K$ is zero-dimensional), then (i) and (ii) are equivalent and imply (iii).

(b) If either $K$ is scattered or $K^{\prime}$ is first countable in itself (e.g., metrizable) and has empty interior in $K$, then the three properties (i), (ii), (iii) are equivalent.

Proof. (a) This follows immediately from Theorem 5.3.

(b) In both cases, the isolated singletons form a pseudo-base for $K$, and hence the equivalence $(i) \Leftrightarrow(i i)$ follows from (a). Since $(i i) \Rightarrow(i i i)$ is obvious, it remains to show the reverse implication. So assume that $K$ satisfies (iii). Let $\emptyset \neq V=\bigcap V_{n}$ where each $V_{n}$ is open in $K$.

If $K$ is scattered, $V$ has an isolated point $v$. There exists an open neighboorhood $U$ of $v$ such that $\{v\}=V \cap U=\bigcap_{n}\left(V_{n} \cap U\right)$. Since $v$ is a $G_{\delta^{-}}$-point of $K$, it is an isolated point of $K$. But then $v \in \operatorname{int} V$.

Now let $K^{\prime}$ be first countable in itself with empty interior in $K$. If $V$ has an isolated point, we proceed as in the first case. If not, then $V=V^{\prime} \subset K^{\prime}$. Fix some $v \in V$. Since $K^{\prime}$ is first countable, $v \in \mathcal{G}_{\delta}(V)$ and hence there exist 
open subsets $G_{n} \subset K(n \in \mathbb{N})$ such that $\{v\}=\left(\bigcap_{n} G_{n}\right) \cap V=\bigcap_{n}\left(G_{n} \cap V_{n}\right)$. It follows that $v \in \mathcal{G}_{\delta}(K)$ but this contradicts (iii).

Now, let us have a look at some other nonreflexive classical spaces. We shall need the following lemmas.

Lemma 5.5. Let $X, Y$ be Banach spaces. If $X$ fails to have (I) then also the direct sums $X \oplus_{\infty} Y$ and $X \oplus_{1} Y$ fail to have (I).

Proof. First, let $Z=X \oplus_{\infty} Y$. Let $\left\{B_{n}\right\} \subset \mathcal{E B}(X)$ be a decreasing sequence such that $\bigcap_{n} B_{n}=B_{X}$ but $\bigcap_{n} B_{n}^{\circ \circ} \neq B_{X^{* *}}$. Since $Z^{*}=X^{*} \oplus_{1} Y^{*}, Z^{* *}=$ $X^{* *} \oplus_{\infty} Y^{* *}$, and the $w^{*}$-topology on $Z^{* *}$ corresponds to the product of the $w^{*}$-topologies of $X^{* *}$ and $Y^{* *}$, the sets $E_{n}:=B_{n} \times B_{Y}(n \in \mathbb{N})$ belong to $\mathcal{E B}(Z)$ and satisfy $\bigcap_{n} E_{n}=B_{X} \times B_{Y}=B_{Z}$ while $\bigcap_{n} E_{n}^{\circ \circ}=\left(\bigcap_{n} B_{n}^{\circ \circ}\right) \times B_{Y^{* *}} \neq$ $B_{X^{* *}} \times B_{Y^{* *}}=B_{Z^{* *}}$. Thus $X \oplus_{\infty} Y$ fails to have (I).

Now, let $Z=X \oplus_{1} Y$. One can proceed in a similar way by using the dual characterization from Proposition 3.3, working in $Z^{*}=X^{*} \oplus_{\infty} Y^{*}$. The details are left to the reader as an easy exercise.

Remark 5.6. A similar statement holds true also for $X \oplus_{p} Y$ (however, we shall not use this fact):

Let $X, Y$ be Banach spaces. If $X$ fails to have (I) then also the direct sum $Z=X \oplus_{p} Y(1<p<\infty)$ fails to have $(I)$.

Let us sketch a proof. Suppose that $X$ fails to have (I). By Proposition 3.3(iv), there exists a sequence $\left\{C_{n}\right\}$ of $w^{*}$-compact, symmetric, convex sets in $X^{*}$ such that $C_{n} \nearrow C$ with $C$ 1-norming, and $\bar{C} \neq B_{X^{*}}$. For $n \in \mathbb{N}$, let us consider the set $E_{n} \subset Z^{*}=X^{*} \oplus_{q} Y^{*}(p, q$ conjugate indices) given by

$$
E_{n}=\left\{\left(x^{*}, y^{*}\right) \in C_{n} \times B_{Y^{*}}:\left\|x^{*}\right\|^{q}+\left\|y^{*}\right\|^{q} \leq 1\right\} .
$$

Then, it is not difficult to see that $\left\{E_{n}\right\}$ is a sequence of $w^{*}$-compact, symmetric, convex sets in $Z^{*}$ such that $E_{n} \nearrow E$ with $E$ 1-norming, and

$$
\bar{E}=\left\{\left(x^{*}, y^{*}\right) \in \bar{C} \times B_{Y^{*}}:\left\|x^{*}\right\|^{q}+\left\|y^{*}\right\|^{q} \leq 1\right\} \neq B_{Z^{*}} .
$$

Hence, by Proposition 3.3(iv), $Z$ fails to have (I).

Lemma 5.7. $\ell_{\infty}^{*}=\ell_{1} \oplus_{1} c_{0}^{\perp}$.

Proof. The proof follows easily by [12, III Proposition 1.2 and III Example 1.4]. However, for the sake of completeness, we include a direct proof for this fact.

Let us consider

$$
F=\left.F\right|_{c_{0}}+F-\left.F\right|_{c_{0}} \in \ell_{\infty}^{*}
$$

and define

$$
H:=\left.F\right|_{c_{0}} \in \ell_{1}, \quad G:=F-\left.F\right|_{c_{0}} \in c_{0}^{\perp} .
$$

Let us prove that $\|F\|=\|H\|+\|G\|$. Let $\varepsilon>0$ and let $x \in B_{\ell_{\infty}}$ be such that $G x \geq\|G\|-\varepsilon$. Since $H \in \ell_{1}$, there exists $n \in \mathbb{N}$ such that $\sum_{k=n+1}^{\infty}|H(k)| \leq \varepsilon$. 
Let us define $y \in B_{\ell_{\infty}}$ as follows: $y(k)=x(k)$, if $k>n ; y(k)=\operatorname{sign}[H(k)]$, if $k \leq n$. Since $G \in c_{0}^{\perp}, G x=G y$ and hence

$\|F\| \geq H y+G y \geq \sum_{k=1}^{n}|H(k)|+\sum_{k=n+1}^{\infty} H(k) y(k)+\|G\|-\varepsilon \geq\|H\|+\|G\|-3 \varepsilon$.

By the arbitrariness of $\varepsilon>0$, we have $\|F\| \geq\|H\|+\|G\|$. The other inequality is obvious.

If $\Gamma$ is an infinite set (always considered in the discrete topology), we denote by $\Gamma_{\infty}$ the Alexandroff one-point compactification of $\Gamma$, and by $\beta \Gamma$ the StoneCech compactification of $\Gamma$.

Proposition 5.8. Let $\Gamma$ be an infinite set.

(a) $\ell_{\infty}(\Gamma)$ fails to have (I).

(b) $c_{0}(\Gamma)$ has property (GI), and hence also (I).

(c) $c(\Gamma):=C\left(\Gamma_{\infty}\right)$ has property (I) if and only if $\Gamma$ is uncountable.

(d) The quotient space $\ell_{\infty}(\Gamma) / c_{0}(\Gamma)$ has property $(I)$.

(e) $\ell_{1}(\Gamma)$ fails to have (I).

(f) $\ell_{\infty}(\Gamma)^{*}$ fails to have $(I)$.

Proof.

(a) First, consider $\Gamma=\mathbb{N}$. It is is well-known that $\ell_{\infty}:=\ell_{\infty}(\mathbb{N})$ is isometric to $C(\beta \mathbb{N})$, and $\beta \mathbb{N}$ is zero-dimensional. Moreover, the remainder $\beta \mathbb{N} \backslash \mathbb{N}=$ $\bigcap_{n \in \mathbb{N}}(\beta \mathbb{N} \backslash\{n\})$ is a nonempty $G_{\delta}$-set with empty interior. By Theorem 5.3, $\ell_{\infty}(\mathbb{N})$ fails to have $(\mathrm{I})$. Now, let $\Gamma$ be uncountable. Then $\ell_{\infty}(\Gamma)$ is clearly isometric to $\ell_{\infty} \oplus_{\infty} \ell_{\infty}(\Gamma)$. The previous case and Lemma 5.5 imply that $\ell_{\infty}(\Gamma)$ fails to have $(\mathrm{I})$.

(b) Let $D \subset c_{0}(\Gamma)^{*}=\ell_{1}(\Gamma)$ be a 1-norming equivalent ball. It is an easy exercise to show that then $D$ contains all $w^{*}$-strongly exposed points of $B_{\ell_{1}(\Gamma)}$, and hence all basic vectors $e_{\gamma}^{*}(\gamma \in \Gamma)$. It follows that $D$ contains also $\overline{\operatorname{conv}}\left\{ \pm e_{\gamma}^{*}: \gamma \in \Gamma\right\}=B_{\ell_{1}(\Gamma)}$. Apply Proposition 3.4.

(c) For $\Gamma$ uncountable, apply Theorem 5.3. For $\Gamma$ countable, observe that $c(\Gamma)$ is separable, and apply Proposition 5.1.

(d) It is well-known that our quotient space is isometric to $C(\beta \Gamma \backslash \Gamma)$. The rest follows from Corollary 5.4(a) and from the fact that $\beta \Gamma \backslash \Gamma$ is a zerodimensional compact space in which every nonempty $G_{\delta}$-set has nonempty interior (for a proof of this last property for $\Gamma=\mathbb{N}$ see [21, Corollary 27]; the same proof works for $\Gamma$ uncountable, too).

(e) If $\Gamma=\mathbb{N}$, the space $\ell_{1}:=\ell_{1}(\mathbb{N})$ is separable but its dual $\ell_{\infty}$ is not. By Theorem 3.6, $\ell_{1}$ fails to have $(\mathrm{I})$. For $\Gamma$ uncountable, the space $\ell_{1}(\Gamma)$ is isometric to the direct sum $\ell_{1} \oplus_{1} \ell_{1}(\Gamma)$. Apply Lemma 5.5.

(f) By (e), $\ell_{1}$ does not have property (I). By Lemma 5.7 and Lemma 5.5, $\ell_{\infty}^{*}$ does not have property (I). 


\section{Property (GI) AND GeOmetry of the DUAL Unit Ball}

Let us recall that the ball topology of a Banach space $X$ is the coarsest (not necessarily Hausdorff) topology $b_{X}$ so that every closed ball is $b_{X}$-closed. This topology was introduced by H.H. Corson and J. Lindenstrauss [6] and studied by several authors (see, e.g., $[10,5,4])$. We say that $X$ has the ball generated property, (BGP) for short, if every closed bounded convex set is $b_{X}$-closed.

It is known (see [10, Theorem 8.3]) that if $X$ has the (BGP) then $X$ is nicely smooth (has property $(\mathrm{N})$, for short), that is, its dual $X^{*}$ has no proper closed subspace $Y$ whose unit ball $B_{Y} \subset B_{X^{*}}$ would be 1-norming for $X$.

It is also well known [11] that $X$ has the (BGP) if and only if the restriction of every element of $X^{*}$ to the unit ball $B_{X}$ is $b_{X}$-continuous. The following sequential version of the (BGP) was introduced and studied in [11].

Definition 6.1 ([11]). A Banach space $X$ has property $(\mathrm{P})$ if the restriction of every bounded linear functional to $B_{X}$ is $b_{X}$-sequentially continuous.

It is clear that if $X$ has the (BGP) then $X$ has property $(\mathrm{P})$; however, the converse implication is false in general Banach spaces (see [11]).

Given a nonempty set $K \subset X^{*}$, a $w^{*}$-slice of $K$ is a set of the form

$$
S(K, x, \alpha)=\left\{x^{*} \in K: x x^{*}>\sup x(K)-\alpha\right\},
$$

where $\alpha>0$ and $x \in X \backslash\{0\}$ is bounded above on $K$. Let us recall the following definitions.

Definition 6.2. Let $K$ be a nonempty subset of $X^{*}$. A functional $x^{*} \in K$ is said to be:

(i) a $w^{*}$-strongly exposed point of $K$ if there exists a nonzero $x \in X$ such that $x x^{*}=\sup x(K)$ and for each norm neighbourhood $V$ of $x^{*}$ there exists $\alpha>0$ such that $S(K, x, \alpha) \subset V$. We denote by $w^{*}-\operatorname{str} \exp (K)$ the set of all $w^{*}$-strongly exposed points of $K$.

(ii) a $w^{*}$-denting point (a $w^{*}-w$-denting point, respectively) of $K$ if for each neighbourhood $V$ of $x^{*}$ in the norm topology (in the weak topology, respectively) there exists a $w^{*}$-slice $S$ of $K$ such that $x^{*} \in S \subset V$. We denote by $w^{*}$-dent $(K)$ the set of all $w^{*}$-denting points of $K$, and by $w^{*}$-w-dent $(K)$ the set of all $w^{*}$-w-denting points of $K$.

(iii) a $w^{*}$-point of continuity (a $w^{*}$-w-point of continuity, respectively) of $K$ if for each neighbourhood $V$ of $x^{*}$ in the norm topology (in the weak topology, respectively) there exists a neighbourhood $W$ of $x^{*}$ in the $w^{*}$-topology such that $W \cap K \subset V$. We denote by $w^{*}$-pc $(K)$ the set of all $w^{*}$-point of continuity of $K$, and by $w^{*}-w$-pc $(K)$ the set of all $w^{*}-w$-points of continuity of $K$. 
The next theorem collects some results, concerning the (BGP) and related properties, contained in [5, Theorem 7], [4, Corollary 4] and [11, Proposition 2.3].

Theorem 6.3. Let $X$ be a Banach space. Let us consider the following conditions.

(a) $\overline{\operatorname{span}}\left(w^{*}-\operatorname{str} \exp \left(B_{X^{*}}\right)\right)=X^{*}$.

(b) $\overline{\operatorname{span}}\left(w^{*}-\operatorname{dent}\left(B_{X^{*}}\right)\right)=X^{*}$.

(c) $\overline{\operatorname{span}}\left(w^{*}-\mathrm{pc}\left(B_{X^{*}}\right)\right)=X^{*}$.

(d) $X$ has the (BGP).

(e) $X$ has property $(\mathrm{N})$.

(f) $X$ has property $(\mathrm{P})$.

Then the following implications hold.

$$
(a) \Rightarrow(b) \Rightarrow(c) \Rightarrow \underset{\substack{\Downarrow \\ \Downarrow}}{(d)} \Rightarrow(e)
$$

Moreover:

- if $X$ is Asplund then we have $(e) \Rightarrow(a)$;

- let $X$ be separable; then all the above conditions are equivalent, and if any of them is satisfied then $X$ is Asplund.

(The fact that if $X$ is separable with (e) or (f) then also $X^{*}$ is separable can be shown as follows. By [11, Proposition $2.3(2)], X$ has $(\mathrm{N})$. Since $\left(B_{X^{*}}, w^{*}\right)$ is compact and metrizable, it contains a dense countable set $S$, which is clearly 1-norming. By property (N), span $S=X^{*}$ which is separable.)

In [4], D. Chen and B.-L. Lin proved the following characterization of the ball generated property.

Proposition 6.4 ([4, Theorem 2]). Let $X$ be a Banach space. Then $X$ has the (BGP) if and only if for each $x_{0}^{*} \in X^{*}$ and each open neighbourhood $W$ of $x_{0}^{*}$ in the norm topology of $X^{*}$ there exist finitely many $w^{*}$-slices $S_{1}, \ldots, S_{n}$ of $B_{X^{*}}$ such that if $x_{i}^{*} \in S_{i}(i=1, \ldots, n)$ then

$$
\operatorname{span}\left(\left\{x_{i}^{*}\right\}_{i=1}^{n}\right) \cap W \neq \emptyset .
$$

In view of the above proposition, we introduce the following definition.

Definition 6.5. Let $X$ be a Banach space, we say that $B_{X}$ has the convex ball generated property, (CBGP) for short, (the weak convex ball generated property, $\left(w\right.$-CBGP), respectively) if for each $x_{0}^{*} \in S_{X^{*}}$ and each open neighbourhood $W$ of $x_{0}^{*}$ in the norm topology (in the weak topology, respectively) of $B_{X^{*}}$ there exist finitely many $w^{*}$-slices $S_{1}, \ldots, S_{n}$ of $B_{X^{*}}$ such that if $x_{i}^{*} \in S_{i}$ $(i=1, \ldots, n)$ then

$$
\operatorname{conv}\left(\left\{x_{i}^{*}\right\}_{i=1}^{n}\right) \cap W \neq \emptyset .
$$


The next theorem shows the relations between properties (GI), (I) and other properties related to the (BGP). In its proof, we use the techniques introduced in $[5,4]$.

Theorem 6.6. Let $X$ be a Banach space. Let us consider the following conditions.
(i) $\overline{\operatorname{conv}}\left(w^{*}-\operatorname{str} \exp \left(B_{X^{*}}\right)\right)=B_{X^{*}}$.
(ii) $\overline{\operatorname{conv}}\left(w^{*}\right.$-dent $\left.\left(B_{X^{*}}\right)\right)=B_{X^{*}}$.
$\left(i i^{\prime}\right) \overline{\operatorname{conv}}\left(w^{*}-w\right.$-dent $\left.\left(B_{X^{*}}\right)\right)=B_{X^{*}}$.
(iii) $\overline{\operatorname{conv}}\left(w^{*}-\operatorname{pc}\left(B_{X^{*}}\right)\right)=B_{X^{*}}$.
$\left(i i i^{\prime}\right) \overline{\operatorname{conv}}\left(w^{*}-w-p c\left(B_{X^{*}}\right)\right)=B_{X^{*}}$.
(iv) $X$ has property (CBGP).
$\left(i v^{\prime}\right) X$ has property ( $w$-CBGP).
(v) $X$ has property (GI).
(vi) $X$ has property (I).

Let the conditions $(a)-(f)$ be as in Theorem 6.3. Then the following implications hold.

$$
\begin{aligned}
& (f) \\
& (a) \Rightarrow(b) \Rightarrow(c) \Rightarrow \stackrel{\Uparrow}{\Uparrow} \quad \Rightarrow \quad(d) \\
& \Uparrow \Uparrow \Uparrow \quad \Uparrow \quad \text { 介 } \\
& (i) \Rightarrow(i i) \Rightarrow(i i i) \Rightarrow(i v) \quad \Uparrow \\
& \left(i i^{\prime}\right) \Rightarrow \underset{\left(i i i^{\prime}\right)}{\Downarrow} \Rightarrow\left(\begin{array}{c}
\Downarrow \\
\left(i v^{\prime}\right)
\end{array} \Rightarrow(v) \Rightarrow(v i)\right.
\end{aligned}
$$

Moreover, if $X$ is Asplund then we have $(e) \Rightarrow(a)$ and $(v) \Rightarrow(i)$, and if $X$ is separable then we have also $(f) \Rightarrow(a)$ and $(v i) \Rightarrow(i)$.

Proof. The following implications are either trivial or contained in Theorem 6.3, except for the implication $(v) \Rightarrow(e)$ which follows easily from Proposition 3.4.

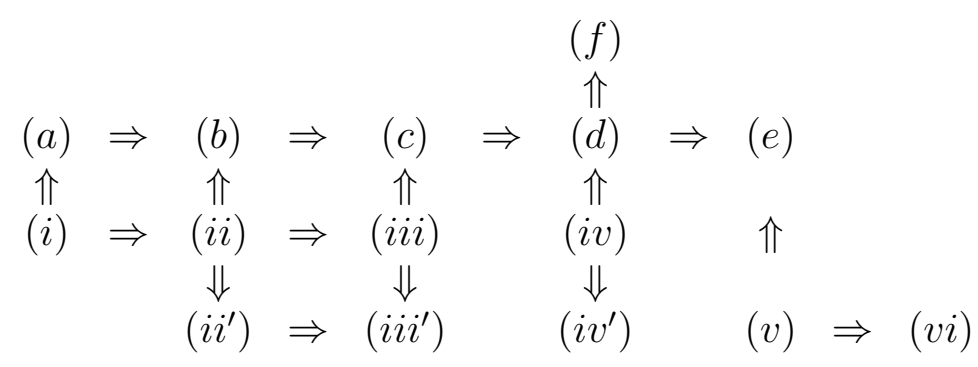

Let us prove that $\left(i i i^{\prime}\right) \Rightarrow\left(i v^{\prime}\right)$, the proof of $(i i i) \Rightarrow(i v)$ is similar. Let $x_{0}^{*} \in S_{X^{*}}$ and let $W$ be an open neighbourhood of $x_{0}^{*}$ in the $w$-topology of $B_{X^{*}}$. Since $\overline{\operatorname{conv}}\left(w^{*}-w\right.$-pc $\left.\left(B_{X^{*}}\right)\right)=B_{X^{*}}$, there exist $x_{1}^{*}, \ldots, x_{n}^{*} \in w^{*}-w$-pc $\left(B_{X^{*}}\right)$ and $a_{1}, \ldots, a_{n} \in(0,1)$ such that $\sum_{i=1}^{n} a_{i}=1$ and $\sum_{i=1}^{n} a_{i} x_{i}^{*} \in W$. Let $W_{i}$ 
$(i=1, \ldots, n)$ be open neighbourhoods of $x_{i}^{*}$ in the $w$-topology of $B_{X^{*}}$ such that $\sum_{i=1}^{n} a_{i} W_{i} \subset W$. Since $x_{1}^{*}, \ldots, x_{n}^{*} \in w^{*}-w$-pc $\left(B_{X^{*}}\right)$, we can and do assume without any loss of generality that $W_{i}(i=1, \ldots, n)$ are open neighbourhoods of $x_{i}^{*}$ in the $w^{*}$-topology of $B_{X^{*}}$. Now, fix $i \in\{1, \ldots, n\}$ and observe that, by the Krein-Milman theorem, there exist $x_{i, 1}^{*}, \ldots, x_{i, n_{i}}^{*} \in \operatorname{ext} B_{X^{*}}$ and $a_{i, 1}, \ldots, a_{i, n_{i}} \in(0,1)$ such that $\sum_{j=1}^{n_{i}} a_{i, j}=1$ and $\sum_{j=1}^{n_{i}} a_{i, j} x_{i, j}^{*} \in W_{i}$. By the Choquet lemma (see [3, Lemma 3.69]), there exist $w^{*}$-slices $S_{i, 1}, \ldots, S_{i, n_{i}}$ of $B_{X^{*}}$ such that $x_{i, j}^{*} \in S_{i, j}\left(j=1, \ldots, n_{i}\right)$ and such that $\sum_{j=1}^{n_{i}} a_{i, j} S_{i, j} \subset W_{i}$. Then

$$
\sum_{i=1}^{n} \sum_{j=1}^{n_{i}} a_{i} a_{i, j} S_{i, j} \subset W
$$

and the proof is concluded.

Let us prove that $\left(i v^{\prime}\right) \Rightarrow(v)$. Let $D \subset B_{X^{*}}$ be a 1-norming closed convex set and suppose on the contrary that there exists $x_{0}^{*} \in S_{X^{*}} \backslash D$, and observe that $W=B_{X^{*}} \backslash D$ is an open neighbourhood of $x_{0}^{*}$ in the $w$-topology of $B_{X^{*}}$. Then there exist $S_{1}, \ldots, S_{n} w^{*}$-slices of $B_{X^{*}}$ such that if $x_{i}^{*} \in S_{i}(i=1, \ldots, n)$ then

$$
\operatorname{conv}\left(\left\{x_{i}^{*}\right\}_{i=1}^{n}\right) \cap W \neq \emptyset .
$$

Since $D$ is 1 -norming, for each $i=1, \ldots, n$, there exists $y_{i}^{*} \in S_{i} \cap D$. Then $\operatorname{conv}\left(\left\{y_{i}^{*}\right\}_{i=1}^{n}\right) \subset D$ and $\operatorname{conv}\left(\left\{y_{i}^{*}\right\}_{i=1}^{n}\right) \cap W \neq \emptyset$, a contradiction and the proof is concluded.

Now, let us assume that $X$ is Asplund and prove that $(v) \Rightarrow(i)$. Since $X$ is Asplund, the Frechét smooth points of $S_{X}$ are dense in $S_{X}$, then it is easy to see that the set $w^{*}$-str $\exp \left(B_{X^{*}}\right)$ is 1-norming and hence that the set

$$
D:=\overline{\operatorname{conv}}\left(w^{*}-\operatorname{str} \exp \left(B_{X^{*}}\right)\right)
$$

is 1-norming. By $(v), D=B_{X^{*}}$ and the proof is concluded. The implication $(e) \Rightarrow(a)$ is contained in Theorem 6.3 .

Let $X$ be separable. If (vi) holds then Theorem 3.6 implies that $X$ is Asplund and satisfies (v). By the already proved part on Asplund spaces, $X$ satisfies (i). Finally, the implication $(f) \Rightarrow(a)$ for $X$ separable is contained in Theorem 6.3.

Let us recall that the duality mapping $D$ of a Banach space $X$ is the multivalued mapping $D: S_{X} \rightarrow 2^{S_{X^{*}}}$, defined by $D(x)=\left\{x^{*} \in S_{X^{*}}: x^{*} x=1\right\}$ $(\neq \emptyset)$. The space $X$ is said to have the Mazur intersection property if every nonempty, closed, bounded, convex subset of $X$ is the intersection of all closed balls in which it is contained.

Corollary 6.7. Let $X$ be a Banach space. Suppose that at least one of the following conditions is satisfied

(i) $X$ is Fréchet smooth. 
(ii) X has the Mazur intersection property.

(iii) $X$ is a polyhedral Banach space.

(iv) The duality mapping $D$ of $X$ is norm-to-weak upper semicontinuous. Then $X$ has property (GI) (and hence property (I)).

Proof. Let us observe that, in each case, we have

$$
\overline{\operatorname{conv}}\left(w^{*} \text {-dent }\left(B_{X^{*}}\right)\right)=B_{X^{*}} .
$$

This is easy to prove in the case $(i)$, and for the cases $(i i),(i i i),(i v)$ it follows by [9, Theorem 2.1], [20, Theorem 2] and the proof of [5, Corollary 8], respectively. Now, the rest follows by Theorem 6.6.

The following proposition shows that properties (BGP) and (GI) (as well as properties $(\mathrm{N})$ and $(\mathrm{I})$ ) do not coincide in general. Given a point $k$ in a compact topological space $K$, recall that $\delta_{k} \in C(K)^{*}$ denotes the corresponding evaluation functional.

Proposition 6.8. There exists a separable Banach space $X$, satisfying property $(\mathrm{N})$ (equivalently, by separability, the (BGP)), such that $X$ does not have property (I) (equivalently, by separability, property (GI)).

Proof. Let us consider the space $X=c=C([1, \omega])$ with the supremum norm ( $\omega$ is the first infinite ordinal). Let $F=\left\{ \pm \delta_{n}+\delta_{\omega} ; n \in \mathbb{N}\right\} \cup\left\{2 \delta_{\omega}\right\}$. It is easy to see that the formula $\|x\|:=\sup |x|(F)=\|x\|_{\infty}+|x(\omega)|$ defines an equivalent norm on $X$, whose dual unit ball is the set $C:=\overline{\operatorname{conv}} w^{*}( \pm F)=\overline{\operatorname{conv}}( \pm F)$ (where the last equality holds by Theorem 2.8 since $\pm F$ is a countable boundary for $C$ ).

We claim that $w^{*}$-str $\exp C=\left\{ \pm \delta_{n} \pm \delta_{\omega}: n \in \mathbb{N}\right\}=: W$. Indeed, on one hand, $\bar{F}^{w^{*}}=F \cup\{0\}$, thus, by Milman's "converse" of the Krein-Milman theorem, all extreme points of $C$ belong to $\pm F \cup\{0\}$ and hence, since $\pm 2 \delta_{\omega} \notin$ $w^{*}$-str $\exp (C)$, we have $w^{*}$-str $\exp (C) \subset W$. On the other hand, it is easy to see that $\pm \delta_{n} \pm \delta_{\omega} \in W$ is strongly exposed by $\pm \mathbf{1}_{\{n\}} \pm \frac{1}{2} \mathbf{1}_{(n, \omega]} \in X$.

Hence, $\overline{\operatorname{span}}\left(w^{*}\right.$-str $\left.\exp (C)\right)=X^{*}$ and

$$
2 \delta_{\omega} \in C \backslash \overline{\operatorname{conv}}\left(w^{*} \text {-str } \exp (C)\right) \neq B_{(X,\|\cdot\|)^{*}} .
$$

By Theorem 6.3 , implication $(\mathrm{a}) \Rightarrow(\mathrm{e})$, the space $(X,\|\cdot\|)$ has property $(\mathrm{N})$. Since $X$ is Asplund, by Theorem 6.6, implication $(\mathrm{v}) \Rightarrow(\mathrm{i}),(X,\|\cdot\|)$ does not satisfy property (GI).

By Proposition 5.8, if $\Gamma$ is an uncountable set then the space $X=c(\Gamma)$ has property (I). Then, by Proposition 5.8, $X$ contains a 1-complemented subspace, namely $c$, that does not have property (I). Hence, property (I) passes neither to subspaces nor to quotients. The next proposition shows that property (I) is not hereditary even if we restrict ourselves to the class of separable Banach spaces. 
Proposition 6.9. There exists a separable Banach space $X$ satisfying property (I) (equivalently, (GI)), and $Y$ a 1-complemented subspace of $X$ such that $Y$ does not have property (I) (equivalently, (GI)).

Proof. Consider the space $X=C([1, \omega])$ with the supremum norm and denote $e_{n}=\mathbf{1}_{\{n\}} \in C([1, \omega]), n \in \mathbb{N}$. Then $X=\mathbb{R} e_{1} \oplus C([2, \omega])$. Let $F=\left\{\delta_{n}: n \geq\right.$ $2\} \cup\left\{\delta_{\omega} \pm \delta_{e_{1}}\right\}$. It is easy to see that $C=\overline{\operatorname{conv}}( \pm F)$ is a $w^{*}$-closed bounded absolutely convex subset of $X^{*}$ with non empty interior, and hence there exists an equivalent norm $\|\cdot\|$ on $X$, whose dual unit ball is $C$.

The points of $F$ are $w^{*}$-strongly exposed for $C$. Indeed, it is easy to see that $\delta_{n}$ is strongly exposed by $e_{n}(n \geq 2)$, and $\delta_{\omega} \pm \delta_{e_{1}}$ is strongly exposed by $\frac{1}{2}\left(\mathbf{1}_{[2, \omega]} \pm e_{1}\right)$. Now, let $D \subset C$ be a 1-norming equivalent ball. Since $D$ must contain all $w^{*}$-strongly exposed points of $C$ (as already observed in the proof of Proposition 5.8(b)), we have $\pm F \subset D \subset C$ and hence $D=C=B_{(X,\|\cdot\|)^{*} \text {. }}$ So $(X,\|\cdot\|)$ has property $(\mathrm{GI})$ by Proposition 3.4 . However, $Y=C([2, \omega])$ is a 1-complemented subspace of $X$ and, by Proposition 5.8(c), it does not have property (GI) (observe that $Y$ is isometric with $c$ ).

It seems natural to ask whether every Asplund space admits an equivalent norm with property (I). The next proposition and the subsequent comment show that the situation for property (I) is analogous to that concerning the Mazur intersection property, and the results obtained depend on set-theoretic axioms (cf. $[13,1])$.

The following proposition is an analogue of [11, Proposition 3.6]; we include a proof for the sake of completeness.

Proposition 6.10. Assuming the continuum hypothesis, there exists a nonseparable Asplund space, namely the Kunen space $C(\mathcal{K})$, admitting no equivalent norm with property (I).

Proof. If $\mathcal{K}$ is the Kunen compact then $C(\mathcal{K})$ is a nonseparable Asplund space since $\mathcal{K}$ is scattered [19, p. 1128]. Moreover, $\left(C(\mathcal{K})^{*}, w^{*}\right)$ is known to be hereditarily seprable (see e.g. [3, p. 638, Remark (ii)]). Now, Theorem 3.6 easily implies that $C(\mathcal{K})$ fails (I), and this is indeed true for any equivalent renorming of $C(\mathcal{K})$.

On the other hand, M. Bačák and P. Hájek [1] proved that, assuming the Martin's Maximum axiom (MM), every Asplund space $X$ of density $\omega_{1}$ admits an equivalent norm with the Mazur intersection property (and hence, by Corollary 6.7, it admits an equivalent norm with property (GI)).

\section{Property (U), property $\left(I^{*}\right)$, and Grothendieck spaces}

Definition 7.1. Let $X$ be a normed linear space. 
(i) We say that $X$ has property (U) if for each sequence $\left\{B_{n}\right\}$ of sets in $\mathcal{E B}(X)$ such that $B_{n} \bar{\nearrow} B_{X}$ one has that $B_{n}^{\circ} \bar{\nearrow} B_{X^{* *}}$.

(ii) We say that $X^{*}$ has property (I*) if for each sequence $\left\{D_{n}\right\}$ of $w^{*}$ compact sets in $\mathcal{E B}\left(X^{*}\right)$ such that $D_{n} \searrow B_{X^{*}}$ one has that $D_{n}^{\circ \circ} \searrow B_{X^{* * *}}$.

Proposition 7.2. Let $X$ be a normed linear space. Then the following assertions are equivalent.

(i) $X$ has property (U).

(ii) For each increasing sequence $\left\{B_{n}\right\}$ in $\mathcal{E B}(X)$ such that $\bigcup_{n \in \mathbb{N}} B_{n} \supset$ $U_{X}$, one has $\bigcup_{n \in \mathbb{N}} B_{n}^{\circ \circ} \supset U_{X^{* *}}$.

(iii) For each increasing sequence $\left\{C_{n}\right\}$ of convex subsets of $X$ such that $\bigcup_{n \in \mathbb{N}} C_{n} \supset U_{X}$ and eventually int $C_{n} \neq \emptyset$, one has $\bigcup_{n \in \mathbb{N}} C_{n}^{\circ \circ} \supset U_{X^{* *}}$.

(iv) $X^{*}$ has property $\left(\mathrm{I}^{*}\right)$.

(v) For each bounded sequence $\left\{x_{n}^{*}\right\}$ in $X^{*}$ whose all $\sigma\left(X^{*}, X\right)$-cluster points are contained in $B_{X^{*}}$, we have that all $\sigma\left(X^{* * *}, X^{* *}\right)$-cluster points (in $X^{* * *}$ ) of $\left\{x_{n}^{*}\right\}$ are contained in $B_{X^{* * *}}$.

Moreover, if $X$ is a Banach space then each of the above conditions is equivalent to

(iii' $\left.^{\prime}\right)$ For each increasing sequence $\left\{C_{n}\right\}$ of convex subsets of $X$ such that $\bigcup_{n \in \mathbb{N}} C_{n} \supset U_{X}$, one has $\bigcup_{n \in \mathbb{N}} C_{n}^{\circ \circ} \supset U_{X^{* *}}$.

Proof. $(i) \Rightarrow(i i)$. Let $\left\{B_{n}\right\}$ be as in (ii). Then the sets $\widetilde{B}_{n}=B_{n} \cap B_{X}$ have the same properties and, moreover, they are all contained in $B_{X}$. By Lemma 2.6, we have $\widetilde{B}_{n} \bar{\nearrow} B_{X}$, and hence also $\left(\widetilde{B}_{n}\right)^{\circ \circ} \bar{\nearrow} B_{X^{* *}}$ by (i). Another application of Lemma 2.6 gives that $U_{X^{* *}} \subset \bigcup_{n \in \mathbb{N}}\left(\widetilde{B}_{n}\right)^{\circ \circ} \subset \bigcup_{n \in \mathbb{N}} B_{n}^{\circ \circ}$.

$($ ii $) \Rightarrow($ iii $)$. Let $\left\{C_{n}\right\}$ be as in (iii). We claim that $0 \in \operatorname{int} C_{p}$ for some $p \in \mathbb{N}$. Indeed, take some $C_{m}$ with nonempty interior and a point $0 \neq x \in$ int $C_{m}$. There exists $p \geq m$ such that $-x \in C_{p}$, but then $C_{p}$ contains the convex set $\operatorname{conv}\left(\{-x\} \cup C_{m}\right)$ whose interior contains 0 , and our claim follows. Thus we can (and do) assume that $0 \in \operatorname{int} C_{1}$. By symmetry of $U_{X}$ and monotonicity of $\left\{C_{n}\right\}$, the sets $B_{n}=C_{n} \cap\left(-C_{n}\right)$ satisfy $\bigcup_{n \in \mathbb{N}} B_{n} \supset U_{X}$. Then $\left\{\bar{B}_{n}\right\}$ is an increasing sequence in $\mathcal{E} \mathcal{B}(X)$, and hence by (ii) we have that $U_{X^{* *}} \subset \bigcup_{n \in \mathbb{N}}\left(\bar{B}_{n}\right)^{\circ \circ}=\bigcup_{n \in \mathbb{N}} B_{n}^{\circ \circ} \subset \bigcup_{n \in \mathbb{N}} C_{n}^{\circ \circ}$.

$($ iii $) \Rightarrow(i v)$. Let $\left\{D_{n}\right\}$ be as in the definition of property $\left(\mathrm{I}^{*}\right)$, and for each $n \in \mathbb{N}$ define $C_{n}={ }^{\circ} D_{n}$. From Lemma 2.6 we obtain that $B_{X} \supset \bigcup_{n \in \mathbb{N}} C_{n} \supset$ $U_{X}$. By (iii), one has $B_{X^{* *}} \supset \bigcup_{n \in \mathbb{N}} C_{n}^{\circ \circ} \supset U_{X^{* *}}$. From Lemma 2.6 again, we obtain that $\bigcap_{n \in \mathbb{N}} D_{n}^{\circ \circ}=\bigcap_{n \in \mathbb{N}}\left(C_{n}^{\circ \circ}\right)^{\circ}=B_{X^{* * *}}$.

$(i v) \Rightarrow(i)$. Let $\left\{B_{n}\right\}$ be as in the definition of property (U) and, for each $n \in \mathbb{N}$, define $D_{n}=B_{n}^{\circ}$. Then $\left\{D_{n}\right\}$ is a sequence of $w^{*}$-compact sets in $\mathcal{E B}\left(X^{*}\right)$. Moreover, by Lemma 2.6, $D_{n} \searrow B_{X^{*}}$. By property $\left(\mathrm{I}^{*}\right)$,

$$
D_{n}^{\circ \circ}=\left(B_{n}^{\circ \circ}\right)^{\circ} \searrow B_{X^{* * *}} \text {. }
$$


Then, by Lemma $2.6, B_{n}^{\circ \circ} \bar{\nearrow} B_{X^{* *} \text {. }}$

$(i v) \Rightarrow(v)$. Let $\left\{x_{n}^{*}\right\} \subset X^{*}$ be a bounded sequence such that the set of all $\sigma\left(X^{*}, X\right)$-cluster points of $\left\{x_{n}^{*}\right\}$ is contained in $B_{X^{*}}$. Let us define

$$
D_{n}:=\operatorname{conv}\left[B_{X^{*}} \cup \overline{\operatorname{conv}}^{\sigma\left(X^{*}, X\right)}\left\{ \pm x_{k}^{*}: k \geq n\right\}\right] .
$$

Then $\left\{D_{n}\right\}$ is a sequence of $w^{*}$-compact sets in $\mathcal{E} \mathcal{B}\left(X^{*}\right)$. It is easy to see that $D_{n} \searrow B_{X^{*}}$. (Indeed, if this is not the case, there exists $y \in S_{X}$ such that $s:=\sup y\left(\bigcap_{n} D_{n}\right)>1$, and hence for each $n \in \mathbb{N}$ there exist $k_{n} \geq n$ and a sign $\theta_{n} \in\{ \pm 1\}$ such that $y\left(\theta_{n} x_{k_{n}}^{*}\right) \geq s$; and this easily leads to a $\sigma\left(X^{*}, X\right)$-cluster point $x^{*}$ of $\left\{x_{n}^{*}\right\}$ such that $\left|y x^{*}\right| \geq s$, and hence $\left\|x^{*}\right\|>1$.) So by property $\left(\mathrm{I}^{*}\right), D_{n}^{\circ} \searrow B_{X^{* * *}}$. Let us observe that

$$
D_{n}^{\circ \circ}=\overline{\mathrm{conv}}^{\sigma\left(X^{* * *}, X^{* *}\right)}\left[B_{X^{*}} \cup\left\{ \pm x_{k}^{*}: k \geq n\right\}\right],
$$

and hence that the set of all $\sigma\left(X^{* * *}, X^{* *}\right)$-cluster points of $\left\{x_{n}^{*}\right\}$ is contained in $\bigcap_{n} D_{n}^{\circ \circ}=B_{X^{* * *}}$.

$(v) \Rightarrow(i v)$. Let $\left\{D_{n}\right\}$ be as in the definition of property $\left(\mathrm{I}^{*}\right)$, and suppose on the contrary that there exists $x^{* * *} \in \bigcap_{n \in \mathbb{N}} D_{n}^{\circ 0}$ such that $\left\|x^{* * *}\right\|>1$. Let $F \in U_{X^{* *}}$ be such that $F x^{* * *}>1$ and define

$$
\mathcal{U}:=\left\{y^{* * *} \in X^{* * *}: F y^{* * *} \geq 1\right\} .
$$

Notice that $\mathcal{U}$ is a neighborhood of $x^{* * *}$ in the $\sigma\left(X^{* * *}, X^{* *}\right)$-topology, and $D_{n}$ is $\sigma\left(X^{* * *}, X^{* *}\right)$-dense in $D_{n}^{\circ \circ}$. Thus for each $n \in \mathbb{N}$ there exists $x_{n}^{*} \in D_{n} \cap \mathcal{U}$. Since $\mathcal{U}$ is $\sigma\left(X^{* * *}, X^{* *}\right)$-closed there exists $G \in \mathcal{U}$, a $\sigma\left(X^{* * *}, X^{* *}\right)$-cluster point of the sequence $\left\{x_{n}^{*}\right\}$. Moreover, for each $n \in \mathbb{N}, D_{n}$ is $\sigma\left(X^{*}, X\right)$-closed and hence the set of all $\sigma\left(X^{*}, X\right)$-cluster points of $\left\{x_{n}^{*}\right\}$ is contained in $\bigcap_{n \in \mathbb{N}} D_{n}=B_{X^{*}}$. Since $\mathcal{U} \cap B_{X^{* * *}}=\emptyset$, we get a contradiction with (v).

To prove the last part of the proposition we have to show that the implication $($ iii $) \Rightarrow\left(\right.$ iii $\left.^{\prime}\right)$ holds for Banach spaces. If $\left\{C_{n}\right\}$ is as in (iii'), by the Baire category theorem we have that eventually $\operatorname{int}\left(\bar{C}_{n}\right) \neq \emptyset$. Since the sequence $\left\{\bar{C}_{n}\right\}$ satisfies the assumptions of (iii), we conclude that $U_{X^{* *}} \subset \bigcup_{n \in \mathbb{N}}\left(\bar{C}_{n}\right)^{\circ \circ}=$ $\bigcup_{n \in \mathbb{N}} C_{n}^{\circ \circ}$, and we are done.

Corollary 7.3. Using the dual characterization from Theorem 7.2, we obtain in the same way as in Corollary 3.5 that a normed linear space $X$ has property $(\mathrm{U})$ if and only if its completion has (U). Therefore, when studying property $(\mathrm{U})$, we can restrict ourselves to Banach spaces.

Let us recall that a Banach space $X$ is a Grothendieck space if each $w^{*}$ convergent sequence in $X^{*}$ is $w$-convergent. A quantitative version of this property was introduced in [2] by defining $c$-Grothendieck spaces for $c \geq 1$. We refer the reader to [2] for the definition and basic properties of such spaces. Let us only remark that if $X$ is $c$-Grothendieck for some $c \geq 1$ then it is Groethendieck (but not vice versa in general). 
The next proposition provides characterizations of property (U) via the intermediate envelope (see Section 2) and the 1-Grothendieck property.

Proposition 7.4. Let $X$ be a Banach space. The following are equivalent.

(i) $X$ has property (U).

(ii) $(\mathrm{I})-\operatorname{env}\left(B_{X}\right)=B_{X^{* *}}$.

(iii) $X$ is 1-Grothendieck.

Proof. $(i i) \Rightarrow(i)$. Suppose that (I)-env $\left(B_{X}\right)=B_{X^{* *}}$. Since the set $E=\bigcup_{n} B_{n}$ is convex and dense in $B_{X}$, we obtain that $B_{X}=\bar{E} \subset(\mathrm{I})-\operatorname{env}(E) \subset B_{X^{* *}}$ (see [14, Remarks 1.1(i)]), and hence (I)-env $(E)=B_{X^{* *}}$ by [14, Remarks 1.1(iii)]. So $E$ (I)-generates $B_{X^{* *}}$ which easily implies that $B_{X^{* *}} \subset \overline{\bigcup_{n} B_{n}^{\circ \circ}}$. Thus (i) holds.

$(i) \Rightarrow(i i)$. Let $\left\{E_{n}\right\}$ be a sequence of sets such that $E_{n} \nearrow B_{X}$. Let us define $C_{n}=\operatorname{conv} E_{n}(n \in \mathbb{N})$. Since $\bigcup_{n} C_{n} \supset U_{X}$ and $X$ has property (U), by (iii') in Proposition 7.2, one has $\bigcup_{n} C_{n}^{\circ \circ} \supset U_{X^{* * *}}$. Since eventually $C_{n}^{\circ \circ}=\overline{\mathrm{conv}} w^{*} E_{n}$ (see Fact 2.1), we obtain

$$
\overline{\bigcup_{n} \overline{\overline{\operatorname{conv}}} w^{*} E_{n}}=B_{X^{* *}} .
$$

Finally, the equivalence $(i i) \Leftrightarrow(i i i)$ follows directly from [2, Proposition 2.2].

Corollary 7.5. Let $X$ be a Banach space. If $X$ has property $(\mathrm{U})$ then $X$ is a Grothendieck space.

The next theorem provides a characterization of reflexivity via property (U). Notice that it also implies that the vice versa in Corollary 7.5 is false in general (however this follows also from [2]).

Theorem 7.6. For a Banach space $X$, the following assertions are equivalent.

(i) $X$ is reflexive.

(ii) Every equivalent norm on $X$ has property (U).

Proof. If $X$ is reflexive then clearly it has property (U), let us prove the reverse implication. Suppose that $X$ is nonreflexive and fix some $\delta \in(0,1 / 2)$. Then, by [17, Theorem 1.13.4] there exist sequences $\left\{x_{n}^{*}\right\} \subset B_{X^{*}}$ and $\left\{x_{k}\right\} \subset B_{X}$ such that

$$
x_{n}^{*} x_{k} \begin{cases}=0 & \text { if } n>k, \\ \geq 1-\delta & \text { if } n \leq k .\end{cases}
$$

Let $x^{* *} \in B_{X^{* *}}$ be a $\sigma\left(X^{* *}, X^{*}\right)$-cluster points of $\left\{x_{k}\right\}$ and $G \in B_{X^{* * *}}$ a $\sigma\left(X^{* * *}, X^{* *}\right)$-cluster point of $\left\{x_{n}^{*}\right\}$. Let us observe that $x^{* *} x_{n}^{*} \geq 1-\delta$, whenever $n \in \mathbb{N}$, and hence that $G x^{* *} \geq 1-\delta$; moreover, for each $k \in \mathbb{N}$, we have $G x_{k}=0$. For $n \in \mathbb{N}$, let us consider the $\sigma\left(X^{*}, X\right)$-compact sets

$$
D_{n}:=\left\{x^{*} \in 2 B_{X^{*}}:\left|x^{*} x_{k}\right| \leq 1, k=1, \ldots, n\right\} .
$$


Clearly, we have

$$
D_{n} \searrow D:=\left\{x^{*} \in 2 B_{X^{*}}:\left|x^{*} x_{k}\right| \leq 1, k \in \mathbb{N}\right\},
$$

and $D$ is the unit ball of an equivalent dual norm. Since $G x_{k}=0$ for each $k \in \mathbb{N}$, we have that $2 G \in \bigcap_{n \in \mathbb{N}} D_{n}^{\circ \circ}$.

Since $x^{* *}$ is a $\sigma\left(X^{* *}, X^{*}\right)$-cluster points of $\left\{x_{k}\right\}$, we have $x^{* *} \in D^{\circ}$ and hence

$$
\sup 2 G\left(D^{\circ}\right) \geq 2 G\left(x^{* *}\right) \geq 2(1-\delta)>1 .
$$

Then $2 G \notin D^{\circ \circ}$ and hence $X^{*}$, endowed with the norm given by $D$, does not have property $\left(\mathrm{I}^{*}\right)$. By Proposition 7.2 , the proof is complete.

In $[14,15]$, O.F.K. Kalenda studied intermediate envelopes of closed convex sets. In particular, [14, Proposition 4.2] characterizes the $C(K)$ spaces $X$ whose unit bidual ball $B_{X^{* *}}$ coincides with the intermediate envelope of the unit ball $B_{X}$. Moreover, [14, Example 4.1], shows that if $X=\ell_{\infty}$ then (I)$\operatorname{env}\left(B_{X}\right)=B_{X^{* *}}$. By Proposition 7.4, we have the following result.

Proposition 7.7. $\ell_{\infty}$ has property $(\mathrm{U})$.

If $X$ is a Banach space and $X^{*}$ has property (I) then clearly $X^{*}$ has also property (I*) (equivalently, $X$ has $(\mathrm{U})$ - see Proposition 7.4 ), but in general properties (I) and $\left(\mathrm{I}^{*}\right)$ for $X^{*}$ do not coincide: consider $X=\ell_{\infty}$ and see Propositions 7.7 and 5.8(f).

It is moreover clear that property (U) does not pass to subspaces; indeed, we can consider $X=\ell_{\infty}$ and any of its non-Grothendieck subspaces. However, the following proposition shows that property (U) passes to quotients.

Proposition 7.8. Let $X$ be a Banach space with property (U) and $Y$ a closed subspace of $X$. Then $Z=X / Y$ has property $(\mathrm{U})$.

Proof. This follows directly from [2, Lemma 3.3] and Proposition 7.4.

By Propositions 7.7, 7.8 and 5.8, we also obtain the following corollary.

Corollary 7.9. $X=\ell_{\infty} / c_{0}$ has properties (I) and (U).

\section{A RÉSUMÉ, OPEN PROBLEMS, AND A REMARK}

In order to highlight connections among the main properties considered by us, we summarize in the following theorem some of their characterizations. While (a) is well-known and easy (see Section 2), the proofs of (b)-(d) are contained in Sections 3 and 7. (As usual, "iff" stands for "if and only if".)

Theorem 8.1. Let $X$ be a Banach space.

(a) $A$ set $A \subset X^{*}$ is 1-norming iff $\overline{\mathrm{conv}} w^{*} A=B_{X^{*}}$. 
(b) $X$ has property (I) iff for every symmetric 1-norming set $A \subset X^{*}$ one has $(\mathrm{I})-\operatorname{env}(A)=B_{X^{*}}$

(c) $X$ has property (GI) iff for every (symmetric) 1-norming set $A \subset X^{*}$ one has $\overline{\operatorname{conv}} A=B_{X^{*}}$.

(d) $X$ has property (U) iff $X^{*}$ has property $\left(\mathrm{I}^{*}\right)$ iff (I)-env $\left(B_{X}\right)=B_{X^{*}}$ iff $X$ is 1-Grothendieck.

A few open problems follow.

Problem 8.2. Does $\ell_{1}(\Gamma)$ admit an equivalent norm with property (I) for some uncountable set $\Gamma$ ?

Problem 8.3. Can a nonreflexive dual space $X^{*}$ have property (I)?

Problem 8.4. Does property (I) imply property $(\mathrm{P})$ ?

Problem 8.5. Suppose that $X$ has property (I). Does there exist a separable subspace $Y$ of $X$ with property (I)?

Remark 8.6. In view of Proposition 7.4, it is easy to see that Theorem 7.6 and [15, Theorem 2.2] are "equivalent", in the sense that one can easily prove any of the two statements by using the other one and Proposition 7.4. Hence, the proof of Theorem 7.6, presented above, can be seen as an alternative proof of $[15$, Theorem 2.2].

\section{Acknowledgement.}

- The authors would like to thank the anonymous referee for a number of useful comments that improved the paper.

- The research of the first author was partially supported by INdAMGNAMPA, Project 2018. The research of the second author was partially supported by INdAM-GNAMPA, Project 2018 and by the University of Milan (Università degli Studi di Milano).

\section{REFERENCES}

[1] M. Bačák and P. Hájek, Mazur intersection property for Asplund spaces, J. Funct. Anal. 255 (2008), 2090-2094.

[2] H. Bendová, Quantitative Grothendieck property, J. Math. Anal. Appl. 412 (2014), 1097-1104.

[3] M. Fabian, P. Habala, P. Hájek, V. Montesinos Santalucía and V. Zizler, Banach space theory. The basis for linear and nonlinear analysis, CMS Books in Mathematics/Ouvrages de Mathématiques de la SMC, Springer-Verlag, New York, 2011.

[4] D. Chen and B.-L. Lin, Ball topology on Banach spaces, Houston J. Math. 22 (1996), 821-833.

[5] D. Chen, Z. Hu and B.-L. Lin, Balls intersection properties of Banach spaces, Bull. Austral. Math. Soc. 45 (1992), 333-342. 
[6] H.H. Corson and J. Lindenstrauss, On weakly compact subsets of Banach spaces, Proc. Amer. Math. Soc. 17 (1966), 407-412.

[7] R. Engelking, General topology. Second edition, Sigma Series in Pure Mathematics 6, Heldermann Verlag, Berlin, 1989.

[8] V.P. Fonf and J. Lindenstrauss, Boundaries and generation of convex sets, Israel J. Math. 136 (2003), 157-172.

[9] J.R. Giles, D.A. Gregory and B. Sims, Characterisation of normed linear spaces with Mazur's intersection property, Bull. Austral. Math. Soc. 18 (1978), 105-123.

[10] G. Godefroy and N.J. Kalton, The ball topology and its applications, Contemp. Math. 85 (1989), 195-237.

[11] A.S. Granero, M. Jiménez Sevilla and J.P. Moreno, Sequential continuity in the ball topology of a Banach space, Indag. Math. 10 (1999), 423-435.

[12] P. Harmand, D. Werner and W. Werner, M-ideals in Banach spaces and Banach algebras, Lecture Notes in Mathematics, 1547. Springer-Verlag, Berlin, 1993.

[13] M. Jiménez Sevilla and J.P. Moreno, Renorming Banach spaces with the Mazur intersection property, J. Funct. Anal. 144 (1997), 486-504.

[14] O.F.K. Kalenda, (I)-envelopes of closed convex sets in Banach spaces, Israel J. Math. 162 (2007), 157-181.

[15] O.F.K. Kalenda, (I)-envelopes of unit balls and James' characterization of reflexivity, Studia Math. 182 (2007), 29-40.

[16] V.L. Klee, Convex sets in linear spaces, Duke Math. J. 18 (1951), 443-466.

[17] R.E. Megginson, An introduction to Banach space theory, Graduate Texts in Mathematics, No. 183, Springer-Verlag, New York, 1998.

[18] W.B. Moors, An elementary proof of James' characterization of weak compactness, Bull. Aust. Math. Soc. 84 (2011), 98-102.

[19] S. Negrepontis, Banach spaces and topology, Handbook of set-theoretic topology, North-Holland, Amsterdam, 1045-1142, 1984.

[20] L. Veselý, Boundary of polyhedral spaces: an alternative proof, Extracta Math. 15 (2000), 213-217.

[21] R.C. Walker, The Stone-Cech compactification, Ergebnisse der Mathematik und ihrer Grenzgebiete, Band 83, Springer-Verlag, New York-Berlin, 1974.

Dipartimento di Discipline Matematiche, Finanza Matematica ed Econometria, Università Cattolica del Sacro Cuore, 20123 Milano, Italy

Email address: carloalberto.debernardi@unicatt.it

Email address: carloalberto.debernardi@gmail.com

Dipartimento di Matematica, Università degli Studi, Via C. Saldini 50, 20133 Milano, Italy

Email address: libor.vesely@unimi.it 